SLAC-PUB-11893

June 2006

\title{
Grand Unification as a Bridge Between String Theory and Phenomenology*
}

\author{
Jogesh C. Pati ${ }^{\dagger}$ \\ Stanford Linear Accelerator Center, Stanford University, Stanford, CA 94309 \\ Department of Physics, University of Maryland, College Park, MD 20740
}

\begin{abstract}
In the first part of the talk, I explain what empirical evidence points to the need for having an effective grand unification-like symmetry possessing the symmetry SU(4)color in $4 \mathrm{D}$. If one assumes the premises of a future predictive theory including gravity - be it string/M theory or a reincarnation - this evidence then suggests that such a theory should lead to an effective grand unification-like symmetry as above in $4 \mathrm{D}$, near the string-GUT-scale, rather than the standard model symmetry. Advantages of an effective supersymmetric $\mathrm{G}(224)=\mathrm{SU}(2)_{L} \times \mathrm{SU}(2)_{R} \times \mathrm{SU}(4)^{c}$ or $\mathrm{SO}(10)$ symmetry in $4 \mathrm{D}$ in explaining (i) observed neutrino oscillations, (ii) baryogenesis via leptogenesis, and (iii) certain fermion mass-relations are noted. And certain distinguishing tests of a SUSY G(224) or SO(10)-framework involving CP and flavor violations (as in $\mu \rightarrow e \gamma, \tau \rightarrow \mu \gamma$, edm's of the neutron and the electron) as well as proton decay are briefly mentioned.

Recalling some of the successes we have had in our understanding of nature so far, and the current difficulties of string/M theory as regards the large multiplicity of string vacua, some comments are made on the traditional goal of understanding vis a vis the recently evolved view of landscape and anthropism.
\end{abstract}

Presented at the Workshop on Einstein's Legacy in The New Millenium, Puri, India, December 15-22, 2005 and also at the symposium "Under The Spell of Physics" at Gerard 't Hooft's 60th birthday celebration at Vlieland, Netherlands, July 14-16, 2006.

*This research was supported by the Department of Energy contract DE-AC02-76SF00515 and by DOE grant No. DE-FG02-96ER41015.

†E-mail: pati@physics.umd.edu; pati@slac.stanford.edu 


\section{Introduction}

The interplay between theory and experiments which probe into the world of the very small and that of the very large has led to remarkable progress over the last five decades. The insights gained in the two worlds have in fact become linked. This is indicated briefly in Chart 1. Studies of the very small have resolved some puzzles of the very large, while those of the very large have constrained our understanding of the very small.

In this talk, I will mainly discuss the world of the very small (down to distances $\sim 10^{-16}$ to $10^{-32} \mathrm{~cm}$ ). In here, progress in the past half of a century has been especially prominent in the quest for unification of fundamental particles and their forces. This had led to the introduction of some unconventional ideas such as those of electroweak unification [1, 2, 3], the color gauge theory along with asymptotic freedom [4] and infrared slavery, quark-lepton unification together with unification of seemingly different electroweak and nuclear forces [5]-[8], baryon-lepton non-conservation, fermion-boson unification [9, 10, and finally a grand unity of all matter and all forces including gravity [11, 12]. These attempts have brought forth in steps the ideas of the standard model of particle physics, grand unification, supersymmetry, supergravity, and finally superstring/M theory with the accompanying notion that space-time has extra dimensions beyond the familiar four $\left(d_{\text {total }}=10 / 11\right)$.

Although I will not discuss much about the world of the very large, let me at least mention a major idea in this context - that of inflation [13] - which evolved in the late 1980s and can go well with the ideas of supersymmetry and grand unification. This idea, presently treated as a paradigm, neatly accounts for some of the puzzles of cosmology, including (i) the observed large-scale gross homogeneity and isotropy of the universe, together with small anisotropies, as well as (ii) almost exact flatness of the universe $\left(\Omega_{\mathrm{TOT}}^{\mathrm{obs}}=1.01_{-0.016}^{+0.009}\left[14\right.\right.$, while $\left.\Omega_{\mathrm{TOT}}^{\text {Inflation }}=1 \pm \mathcal{O}\left(10^{-4}\right)\right)$. Inflation in turn requires physics of the very small, beyond that of the standard model, that can link very well with the physics of grand unification. In the process, it also serves to efficiently dilute, what would have been an embarrassment for grand unification, that is super heavy topological structures such as magnetic monopoles. ${ }^{a}$ I will mention in Section 7 a likely link between inflation and grand unification in connection with baryogenesis via leptogenesis, leading to $n_{b} / s \sim 10^{-10}$.

Returning to the various attempts of unification listed above, string/M theory, contemplating physics at truly short distances $\left(\sim 10^{-32} \mathrm{~cm}\right)$, is the most ambitious of all in that it offers the scope for unifying all matter and all forces including gravity and simultaneously providing a good quantum theory of gravity. But it is the least understood of all. As a result, it is yet to make contact with the real world. ${ }^{\mathrm{b}}$ The difficulty is that it exhibits, at the present level of exploration, a very large degeneracy in its vacuum solutions without a guiding principle to select among them. This

\footnotetext{
${ }^{a}$ This is assuming that inflation occurred after the monopole production.

b It does make a non-trivial prediction, however, that is the existence of gravity.
} 


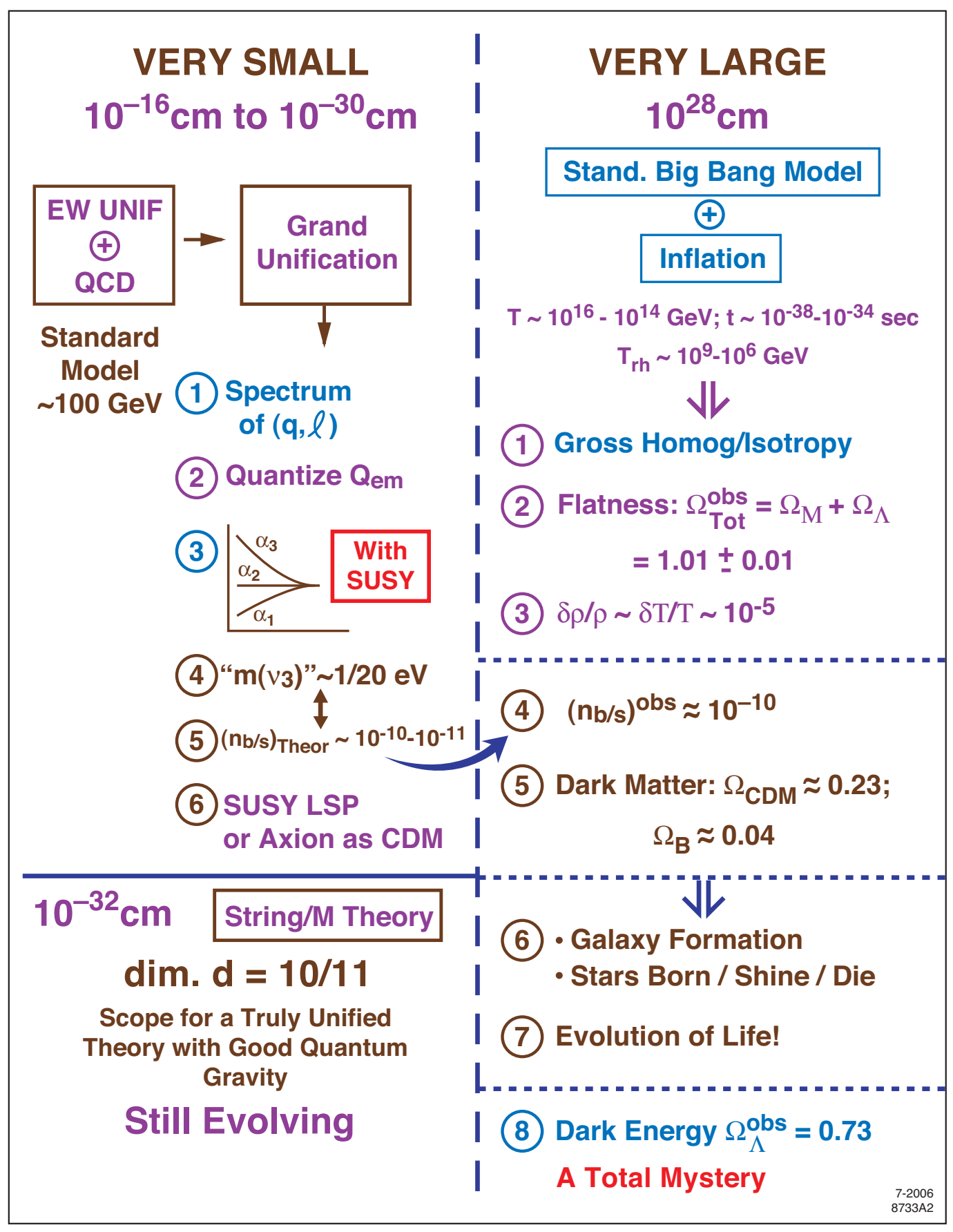

Chart 1: Some insights gained in the world of the very small and that of the very large, which have in fact become linked. 
situation may hopefully be resolved by a better understanding of the theory and/or quite possibly by the introduction of some radically new ingredients, which may provide selectivity.

Meanwhile, it is encouraging that there exist string/M theory solutions in $4 \mathrm{D}$, which, although by no means unique, are at least semi-realistic in that they possess SM-like or grand unification-like gauge symmetries with three families. This feature, together with the scope for a complete unity offered by the string/M theory, raises the hope (for many including me) that string/M theory may well evolve, possibly with the introduction of new ingredients, so as to describe nature in a predictive manner, explaining some of its puzzles. I will briefly comment on these features at the end.

I will first explain in the next few sections why there is a need on empirical grounds to have an effective grand unification-like symmetry in $4 \mathrm{D}$, that too of a special class, near the string-scale of $10^{17}-10^{18} \mathrm{GeV}$. On this basis, I will argue at the end that an underlying unified theory including gravity - be it string/M theory or a reincarnation - as and when it evolves so as to be predictive - should lead to such a grand unification-like symmetry in $4 \mathrm{D}$, rather than the SM-symmetry. That in turn would serve as a very useful bridge between string theory and phenomenology, in accord with observations.

While the contact between string theory and phenomenology is not yet in sight, fortunately the standard model (SM) of particle physics and even the ideas of grand unification and low-energy supersymmetry lend themselves amply to experimental verification; and they also serve to explain some of the intriguing puzzles of nature.

The SM of particle physics, comprising the notions of electroweak unification and QCD, is now in excellent agreement with experiments at least up to energies of order $100 \mathrm{GeV}$ (i.e. distance scales $\sim 10^{-16} \mathrm{~cm}$ ). There are, however, a few observations such as neutrino oscillations (see discussion in Section 3), baryon asymmetry, the need for inflation and that for cold dark matter (not to mention dark energy), which clearly call for new physics beyond the SM.

The next step in the unification-ladder is the hypothesis of grand unification [5]-8], which proposes an underlying unity of quarks and leptons and of their three gauge forces - weak, electromagnetic and strong. Even though the main arena of grand unification lies at superhigh energies $\left(\sim 10^{16} \mathrm{GeV}\right)$ and thus at very short distances $\left(\sim 10^{-30} \mathrm{~cm}\right)$, where the underlying unity mentioned above is presumed to prevail, fortunately it does make several intriguing predictions for low energy physics, some of which have been probed experimentally. These include: (i) first and foremost, the quantum numbers of the members of a family, (ii) quantization of electric charge, (iii) gauge coupling unification and thereby the weak angle $\sin ^{2} \theta_{W}$, (iv) for a certain class of unification symmetries (though not for all) neutrino oscillations, as observed, and (v) baryon excess via lepton excess. These probings lend strong support in favor of the basic idea of grand unification. As we will see, they even serve to select out the nature of the underlying symmetry. Grand unification combined with supersymmetry (mentioned below) offers further tests by which it can be falsified or further vindicated. 
These include predictions for processes such as $\mu \rightarrow e \gamma, \tau \rightarrow \mu \gamma$, EDM transitions of the neutron and the electron, and last but not least, proton decay. I will mention some of these predictions towards the end.

Supersymmetry is an idea that evolved in parallel with that of grand unification in the early 1970s 9]. It proposes a symmetry between fermions and bosons. Remarkably enough, it is needed for consistency of string theory. And, quite independently, there is a compelling reason to believe that supersymmetric partners of quarks and leptons and of the other SM particles should exist with masses near the TeV-scale, because such a picture seems (in my opinion) to be the only natural and viable explanation we have so as to avoid large quantum corrections to the Higgs mass and thereby unnatural extreme fine tuning ${ }^{c}$. As a byproduct, such a SUSY spectrum leads to a dramatic meeting of the three gauge couplings [15, 8] - a feature that in turn supports the hypotheses of grand unification and low energy SUSY. Such a SUSY spectrum also provides a natural candidate for cold dark matter that is needed to account for observations involving the very large. Fortunately SUSY particles with masses near the TeV-scale can be searched for at the forthcoming LHC.

Even if the ideas of grand unification and low-energy supersymmetry are on the right track, it is natural to believe that they arise from an underlying unified theory that includes gravity and provides good quantum theory of gravity. The prime candidate in this regard is the string/M theory, which is formulated in 10/11 dimensions. The questions then arise: (1) should such a higher dimensional theory, upon compactification of the extra dimensions, lead to an effective theory in 4D near the GUT/string-scale $\left(\sim 10^{16}-10^{18} \mathrm{GeV}\right)$ that is SM-like, or grand unification-like? And (2), if it is the latter, which of the alternative symmetries - $\mathrm{SU}(5)$ [7, or $\mathrm{SO}(10)$ [19, or an effective symmetry like $\mathrm{G}(224)=\mathrm{SU}(2)_{L} \times \mathrm{SU}(2)_{R} \times \mathrm{SU}(4)^{c}[6]$, or $[\mathrm{SU}(3)]^{3}[20$, or flipped $\mathrm{SU}(5) \times \mathrm{U}(1)$ [21] - is preferred by data, if any?

I first discuss (in Sections 2-6) why several empirical observations strongly suggest the need for new physics beyond the SM in 4D, of a nature that impressively matches with the predictions of grand unification. Furthermore, I will note why certain observations such as (i) neutrino oscillations, (ii) the success of leptogenesis as a means for baryogenesis, and (iii) that of certain fermion mass relations, even serve to select out the route to higher unification based on the symmetry SU(4)-color in 4D. This in turn suggests, as the simplest possibility, that the effective symmetry in 4D near the string/GUT-scale should minimally be $\mathrm{G}(214)=\mathrm{SU}(2)_{L} \times \mathrm{I}_{3 R} \times \mathrm{SU}(4)^{c} \supset \mathrm{G}(213)$, or

${ }^{\mathrm{c}}$ Without going into details, I should mention, however, that low-energy supersymmetry, in general, poses some generic problems, including: (i) large flavor changing neutral current (FCNC) transitions; (ii) gravity-induced $d=4$ rapid proton decay, and (iii) typically large edm's of the neutron and the electron. However, there also exist well-motivated ideas on SUSY-breaking such as gaugino or gauge-mediation (see e.g. M. Luty 16 for a review) which neatly resolve the FCNC problem (i). There also exist simple resolutions of (ii) and (iii) based on symmetries; see e.g. Pati [17], where symmetries of explicit three family string-derived solutions [18 adequately address (ii). Thus, on balance, low-energy supersymmetry remains a well-motivated and viable idea needing direct test at the LHC. 
$\mathrm{G}(224)$, or a simple group like $\mathrm{SO}(10)$ or $\mathrm{E}_{6}$ [22], all of which possess $\mathrm{SU}(4)$-color, as opposed to alternative symmetries like $\mathrm{SU}(5)$ or $[\mathrm{SU}(3)]^{3}$, which do not.

In Sections 2 and 3, I will recall certain desirable features of symmetries containing $\mathrm{SU}$ (4)-color. In Sections 4-8, I will discuss briefly some results on CP and flavor violations, and last but not least proton decay which arise in the same context.

In the concluding part of my talk, covered in Section 10, I will express a point of view promoting the search for an underlying unified theory-be it string/M theory or a reincarnation - that would describe nature in a predictive manner and would explain some of its presently unexplainable features. In this regard, I will provide a perspective inspired by the successes which we have had over the last 400 years by way of our understanding of nature. They include first and foremost the successes of the two theories of relativity and quantum mechanics. In the present context, they also include the successes of the ideas of the standard model, grand unification and inflation. Each of these have aided (in varying degrees) to our understanding of nature. At the same time, interestingly enough, each of these have provided certain ingredients that are crucial to the origin of life. Based on these features, I would express a view at the end that leans towards the traditional approach to understanding as opposed to the recently evolved view of landscape, combined with anthropism.

\section{The Need for Grand Unification with SU(4)-Color in 4D}

The idea of grand unification [5] - 7] was motivated on aesthetic grounds to achieve a) a unification of quarks and leptons, and b) a unity of the electroweak and strong forces. Simultaneously it was inspired by the desire to explain c) the observed quantum numbers of the members of a family, and d) quantization of electric charge. Over the years, the evidence building up in favor of grand unification (including both old and new) has become strong [23]. It includes:

1. First and foremost, the spectrum of quarks and leptons in a family;

2. Quantization of electric charge;

3. $Q_{e^{-}} / Q_{p}=-1$;

4. The dramatic meeting of the three gauge couplings;

5. Equally dramatic, neutrino oscillations [24]-[26] with $\sqrt{\Delta m^{2}(\nu)_{23}} \approx 1 / 20 \mathrm{eV}$;

6. The success of the two fermion mass relations:

(a) $m_{b}\left(M_{\mathrm{GUT}}\right) \approx m_{\tau}$, and

(b) $m\left(\nu_{\text {Dirac }}^{\tau}\right) \approx m_{\text {top }}\left(M_{\mathrm{GUT}}\right)($ needed for seesaw $)$; and

7. Successful baryogenesis via leptogenesis leading to [27, 28, $Y_{B} \sim 10^{-10}$. 
Of these, the first three are old. They served as the driving motivations for grand unification. The last four pieces of evidence have emerged subsequently.

As mentioned before, the meeting of the three gauge couplings, based on accurate LEP data, occurs if one assumes low-energy supersymmetry [15]. Such a meeting thus provides a strong support both for the ideas of grand unification being operative in $4 \mathrm{D}$ (or for an underlying theory like string theory ensuring coupling unification irrespective of a grand unification symmetry in $4 \mathrm{D}$, see below) and low-energy supersymmetry. The meeting of the gauge couplings extrapolated from low momenta occurs at a scale $M_{\mathrm{GUT}} \approx 2 \times 10^{16} \mathrm{GeV}$. As we will see, this scale $M_{\mathrm{GUT}}$ plays a crucial role in our understanding of the tiny neutrino masses as well as phenomena such as leptogenesis in the context of inflation.

While the first four features (1)-(4) provide strong support, on empirical grounds, in favor of grand unification, they leave open the question of the choice of the effective symmetry $G$ in $4 \mathrm{D}$ near the GUT-scale. In particular, they do not make a sharp distinction between the alternatives of (i) $\mathrm{SU}(5)$, (ii) $\mathrm{SO}(10)$, (iii) $E_{6}$, (iv) $[\mathrm{SU}(3)]^{3}$, or (v) a string-derived semi-simple group like $\mathrm{G}(224)$, with coupling unification being ensured in this case by string theory at the string scale (see remarks below), or (vi) flipped $\mathrm{SU}(5) \times \mathrm{U}(1)$. Of these, the symmetries $\mathrm{G}(224), \mathrm{SO}(10)$ and $E_{6}$ possess the symmetry $\mathrm{SU}(4)$-color, while $\mathrm{SU}(5),[\mathrm{SU}(3)]^{3}$ and flipped $\mathrm{SU}(5) \times \mathrm{U}(1)$ do not.

I would argue in this section and the next that the last three features, involving: (5) Neutrino oscillations, (6) The success of the two mass relations 1(a) and 1(b), and $(7)$ The success of baryogenesis via leptogenesis, clearly suggest that the effective symmetry $G$ in $4 \mathrm{D}$ should possess the symmetry $\mathrm{SU}(4)$-color. I will mention in a moment the common advantages shared by $\mathrm{SO}(10)$ and a string-derived $\mathrm{G}(224)$ symmetry as well as the distinctions between them.

\subsection{The Family Multiplet Structure of G(224)}

To see the need for having SU(4)-color as a component of the higher gauge symmetry, it is useful to recall the family-multiplet structure of $\mathrm{G}(224)$, which is retained by $\mathrm{SO}(10)$ as well. The symmetry $\mathrm{G}(224)=\mathrm{SU}(2)_{L} \times \mathrm{SU}(2)_{R} \times \mathrm{SU}(4)^{c}$, subject to leftright discrete symmetry which is natural to $\mathrm{G}(224)$, organizes members of a family into a single left-right self-conjugate multiplet $\left(\mathrm{F}_{\mathrm{L}}^{\mathrm{e}} \oplus \mathrm{F}_{\mathrm{R}}^{\mathrm{e}}\right)$ given by $[\underline{6}$ :

$$
\mathrm{F}_{\mathrm{L}, \mathrm{R}}^{\mathrm{e}}=\left[\begin{array}{cccc}
\mathrm{u}_{\mathrm{r}} & \mathrm{u}_{\mathrm{y}} & \mathrm{u}_{\mathrm{b}} & \nu_{\mathrm{e}} \\
\mathrm{d}_{\mathrm{r}} & \mathrm{d}_{\mathrm{y}} & \mathrm{d}_{\mathrm{b}} & \mathrm{e}^{-}
\end{array}\right]_{\mathrm{L}, \mathrm{R}}
$$

The multiplets $\mathrm{F}_{\mathrm{L}}^{\mathrm{e}}$ and $\mathrm{F}_{\mathrm{R}}^{\mathrm{e}}$ are left-right conjugates of each other transforming respectively as $(\mathbf{2}, \mathbf{1}, 4)$ and $(\mathbf{1}, \mathbf{2}, 4)$ of $\mathrm{G}(224)$; likewise for the muon and the tau families. Note that each family of G(224), subject to left-right symmetry, must contain sixteen two-component objects as opposed to fifteen for $\mathrm{SU}(5)$ or the standard model. While the symmetries $S U(2)_{L, R} \subset G(224)$ treat each column of $\mathrm{F}_{\mathrm{L}, \mathrm{R}}^{\mathrm{e}}$ as doublets, the sym-

metry $\mathrm{SU}(4)$-color unifies quarks and leptons by treating each row of $\mathrm{F}_{\mathrm{L}}^{\mathrm{e}}$ and $\mathrm{F}_{\mathrm{R}}^{\mathrm{e}}$ as a 
quartet. Thus both $S U(4)$-color and $S U(2)_{R}$ predict the existence of the right-handed neutrino as an essential member of each family, with non-trivial $S U(4)^{c}$ and $S U(2)_{R}$ quantum numbers [6]. In particular, $\mathrm{SU}(4)$-color treats the left and right-handed neutrinos $\left(\nu_{L}^{e}\right.$ and $\left.\nu_{R}^{e}\right)$ as the fourth color-partners of the left and right-handed up quarks $\left(u_{L}\right.$ and $\left.u_{R}\right)$ respectively; likewise for the $\mu$ and the $\tau$ families. This is why $\mathrm{SU}(4)$-color leads to some very desirable fermion mass relations for the third family (i.e. both Eqs. 1(a) and 1(b)) that are empirically favored.

An accompanying characteristic of $S U(4)$-color is that it also introduces $B-L$ as a local symmetry. Now, using observed gauge coupling unification, one can argue that $\mathrm{B}-\mathrm{L}$ and thus $\mathrm{SU}(4)$-color-be it part of $\mathrm{SO}(10)$ or a string-derived $\mathrm{G}(224)$ symmetry - should break spontaneously near the GUT-scale rather than near the Planck or an intermediate scale. Thus, $M_{\mathrm{B}-\mathrm{L}} \sim M_{\mathrm{GUT}}$, where $M_{\mathrm{B}-\mathrm{L}}$ denotes the (B$\mathrm{L}$ )-breaking scale. That in turn says that the Majorana mass of the RH neutrino (at least the heaviest one) which necessarily breaks B-L, should be near the GUT-scale (see Section 3) rather than being much heavier $\left(\sim M_{\text {Planck }}\right)$ or much lighter than the GUT-scale ( $\sim 1$ to $10 \mathrm{TeV}$, say). As we will see in Section 3, such a constraint on the Majorana mass of the RH neutrino, which would not exist if $\mathrm{B}-\mathrm{L}$ were not a part of the gauge symmetry (as in SU(5)), plays a crucial role in yielding the desired mass of the light LH neutrino as well as in providing successful baryogenesis via leptogenesis.

\subsection{Advantages of $\mathrm{G}(224)$}

The symmetry $\mathrm{G}(224)$, supplemented by L-R discrete symmetry which is natural to $\mathrm{G}(224)$, brings a host of desirable features. Including some of those mentioned above which served as motivations for grand unification, they are:

(i) Unification of all sixteen members of a family within one left-right self-conjugate multiplet with a neat explanation of their quantum numbers;

(ii) Quantization of electric charge;

(iii) $Q_{e^{-}} / Q_{p}=-1$;

(iv) Quark-lepton unification through SU(4)-color;

(v) Conservation of parity at a fundamental level [29]; ${ }^{\mathrm{d}}$

(vi) RH neutrino as a compelling member of each family that is now needed for seesaw and leptogenesis;

\footnotetext{
${ }^{\mathrm{d}}$ It appears aesthetically attractive to assume that symmetries like Parity (P), Charge Conjugation (C), CP and Time Reversal ( $\mathrm{T}$ ) break only spontaneously like the gauge symmetries. While such a preference a priori is clearly subjective, and is not respected by symmetries such as $\mathrm{SU}(5)$, observations of neutrino oscillations and the likely need for leptogenesis, suggesting the existence of $\nu_{R}$ 's a la $\mathrm{SU}(4)$-color and $\mathrm{SU}(2)_{L} \times \mathrm{SU}(2)_{R}$, seem to go well with the notion of exact conservation of parity at a fundamental level.
} 
(vii) B-L as a local symmetry. It has been realized eventually that this is needed to protect $\nu_{R}$ 's from acquiring Planck scale masses and to set (for reasons noted above) $M\left(\nu_{R}^{i}\right) \propto M_{B-L} \sim M_{\mathrm{GUT}}$, both crucial to seesaw and leptogenesis;

(viii) The rationale for the two successful mass-relations 1(a) and 1(b), the first $\left(m_{b}\left(M_{\mathrm{GUT}}\right) \approx m_{\tau}\right)$ being empirically successful and the second $\left(m\left(\nu_{\text {Dirac }}^{\tau}\right) \approx\right.$ $\left.m_{\mathrm{top}}\left(M_{\mathrm{GUT}}\right)\right)$ being a crucial ingredient for the success of the seesaw.

These eight features constitute the hallmark of G(224). Historically, all the ingredients underlying these features, and explicitly (i)-(vii), including the RH $\nu$ 's, $\mathrm{B}-\mathrm{L}$ and $\mathrm{SU}(4)$-color, were introduced into the literature only through the symmetry $\mathrm{G}(224)$ [6] this was well before $\mathrm{SO}(10)$ or (even) $\mathrm{SU}(5)$ appeared. Any simple or semi-simple group that contains $\mathrm{G}(224)$ would of course naturally possess these features. So does therefore $\mathrm{SO}(10)$, which is the smallest simple group containing $\mathrm{G}(224)$. In fact, all the advantages of $S O(10)$, which distinguish it from $S U(5)$ and are now needed to understand neutrino oscillations as well as baryogenesis via leptogenesis, arise entirely through the symmetry G(224). $\mathrm{SO}(10)$ being the smallest extension preserves even the family-multiplet structure of $\mathrm{G}(224)$ without needing additional fermions (unlike $\left.E_{6}\right)$. The L-R conjugate 16-plet $=\left(F_{L} \oplus F_{R}\right)$ of $\mathrm{G}(224)$ precisely corresponds to the spinorial $\mathbf{1 6}=\left(F_{l} \oplus\left(F_{R}\right)^{c}\right)$ of $\mathrm{SO}(10)$. Furthermore, with $\mathrm{SU}(4)$-color being vectorial, $\mathrm{G}(224)$ is anomaly-free; $\mathrm{SO}(10)$ is anomaly-free as a group.

As we will see, the last three features (vi), (vii) and (viii) are strongly favored by observations; and they in turn clearly favor the class of symmetries possessing $\mathrm{SU}(4)$ color (like $\mathrm{G}(214), \mathrm{G}(224), \mathrm{SO}(10)$ and $E_{6}$ ) over those which do not. For instance $\mathrm{SU}(5)$, devoid of $\mathrm{SU}(4)$-color, does not provide the ingredients of (vi) RH neutrino, (vii) B-L, and (viii) the mass relation 1(b), though it does provide 1(a). As a result it does not have a natural setting for understanding neutrino masses and implementing baryogenesis via leptogenesis (see discussion in Section 3 and especially footnote $\mathrm{g}$ ).

\subsection{Similarities and Distinctions Between $\mathrm{SO}(10)$ Versus a String-Derived $\mathrm{G}(224)$ in $4 \mathrm{D}$}

In the context of an underlying unified theory like string/M theory defined in higher dimensions $(d=10 / 11)$ the question arises: should the four-dimensional symmetry obtained through compactification of the extra dimensions contain $\mathrm{SO}(10)$ or just G(224)? Quite clearly both share the advantages (i)-(viii) primarily because both contain SU(4)-color and quantize electric charge.

Distinctions between them arise, however, as regards the issues of (i) gauge coupling unification, (ii) the so-called doublet-triplet splitting problem, and (iii) proton decay. I have discussed these issues in more detail elsewhere [30].

Briefly speaking, for the case of a string-derived G(224)-solution [31], coupling unification $\left(g_{2 L}=g_{2 R}=g_{4}\right)$ can hold at the string scale $M_{s t} 32$ through the constraints of string theory even though $\mathrm{G}(224)$ is semi-simple. As mentioned before, 
one would then need to assume that the string-scale is not far above the conventional GUT-scale $\left(M_{\mathrm{st}} \approx(2-3) M_{\mathrm{GUT}}\right.$, say, with $\left.M_{\mathrm{GUT}} \approx 2 \times 10^{16} \mathrm{GeV}\right)$ where $\mathrm{G}(224)$ should break to $G(213)$ to explain observed gauge coupling unification [33. While such a possibility can well arise in the string-context [34, an $\mathrm{SO}(10)$-solution would have an advantage in this regard in that it would ensure gauge coupling unification at the GUT-scale regardless of the gap between the string and the GUT-scales.

At the same time, as noted by several authors (see e.g. Ref. [18 and [31]), a $\mathrm{G}(224)$-solution in 4D would, however, have a distinct advantage over an SO(10)solution as regards the problem of doublet-triplet splitting. This is because, for a $\mathrm{G}(224)$ solution, the undesired color-triplets, which could induce rapid proton decay, can be naturally projected out through the process of string compactification. On the other hand, for an $\mathrm{SO}(10)$-solution the entire 10-plet must exist in 4D. One must then invoke a suitable doublet-triplet splitting mechanism in 4D which would make the color-triplets in $10_{H}$ superheavy, while keeping the $\mathrm{SU}(2)$-doublets in $10_{H}$ light. Such a mechanism can be constructed in 4D [35]; but it is not clear whether such a mechanism can in fact emerge consistently from a string theory.

Given the relative advantages of $\mathrm{SO}(10)$ and a string-derived $\mathrm{G}(224)$ over each other as four-dimensional symmetries, and the fact that the possible disadvantage in each case has at least a plausible solution, I have expressed elsewhere 30] that it is prudent to keep an open mind at present towards both, especially because they share the advantages (i)-(viii) and lead essentially to the same predictions for fermion masses, neutrino oscillations, and leptogenesis (see Sections 4 and 6). I will therefore use them interchangeably for most purposes. I will mention briefly in Sections 5, 6 and 8 that the two cases can be distinguished empirically by phenomena involving $\mathrm{CP}$ and flavor violations as well as proton decay.

\section{The SuperK Scale, Seesaw and SU(4)-color}

Atmospheric neutrino oscillation discovered at SuperK [24] yields a mass-scale $\sqrt{\Delta m^{2}(\nu)_{23}} \approx 1 / 20 \mathrm{eV}$, with an oscillation angle $\sin ^{2} 2 \theta_{23}^{\nu} \approx 0.92-1$. By using WMAP and other astronomical data, one also knows that $\sum m\left(\nu_{i}\right)<0.68 \mathrm{eV}$ [14]. In other words, even the heaviest among the three light neutrinos is lighter than 0.68 $\mathrm{eV}$. These non-vanishing but tiny neutrino masses seem to be extraordinarily small compared with the masses of the charged fermions. For example, comparing ratios of masses of fermions in the same family, which I think is more appropriate for the purpose, ${ }^{\mathrm{e}}$ we see that the mass-hierarchies among the charged fermions are of order $1 / 10$ - 1/100 (e.g. $m_{b} / m_{t} \sim 1 / 45 ; m_{\tau} / m_{t} \sim 1 / 70 ; m_{s} / m_{c} \sim 1 / 8, m_{\mu} / m_{c} \sim 1 / 3$ ). By contrast, comparing masses of neutrinos with the heaviest charged fermion in the

\footnotetext{
eSome may wish to compare the lightest in the first family with the heaviest in the third family. Even here, the ratio $m_{\nu_{e}} / m_{\text {top }} \lesssim(1 / 10 \mathrm{eV}) /(120 \mathrm{GeV}) \sim 10^{-12}$ is much smaller than $m_{e} / m_{\text {top }} \sim$ $10^{-5}$.
} 
same family, we see that the ratios

$$
\left[m\left(" \nu_{\tau} "\right) / m_{\text {top }}\right]_{\mathrm{obs}} \sim(1 / 10-1 / 2) \mathrm{eV} / 120 \mathrm{GeV} \sim 10^{-12}-10^{-11},
$$

and likewise $m\left(\right.$ " $\nu_{\mu}$ ") $/ m_{\mu} \lesssim 10^{-10}-10^{-9}$ are way too small compared with the corresponding ratios of the masses of the charged fermions in the same family, as mentioned above.

While we do not really understand at present the latter i.e. intra- and interfamily mass-hierarchies among the charged fermions, the extraordinarily small neutrino masses seem to suggest that some characteristically new physics is at play in ensuring the tiny neutrino masses. ${ }^{\mathrm{f}}$ In short, ratios such as $m\left(\right.$ " $\nu_{\tau}$ ") $/ m_{\text {top }} \sim 10^{-12}$ pose a major puzzle. As we will see, understanding this ratio and thus the superK mass-scale would shed much light on the nature of the underlying symmetry.

First of all, one can argue that the superK scale clearly calls for physics beyond those of the SM, even with allowance for quantum gravity. This is because, with only LH neutrinos in the SM, even if one allows for lepton number violation through quantum gravity-effects and thereby permits [36] an effective interaction of the form $\kappa L L \phi_{H} \phi_{H} / M_{p l}$, it would yield a Majorana mass-term for $\nu_{L}$ given by $\kappa \nu_{L}^{T} \nu_{L}\left\langle\phi_{H}\right\rangle^{2} / M_{p l}$, and thus a Majorana mass $m\left(\nu_{L}\right)=\kappa\left\langle\phi_{H}\right\rangle^{2} / M_{p l} \approx$ $\kappa(250 \mathrm{GeV})^{2} / 2 \times 10^{18} \mathrm{GeV} \approx \kappa\left(3 \times 10^{-5} \mathrm{eV}\right)$. This is too small by a factor of $10^{3}$ compared to the SuperK value (one of course does not want $\kappa$ to be much greater than one). The situation, however, changes dramatically, as discussed below, when there is a RH neutrino (in addition to the LH one) in the context of a GUT-like symmetry like $\mathrm{G}(224)$ or $\mathrm{SO}(10)$.

In a theory with $\mathrm{RH}$ neutrinos as an essential member of each family, and with spontaneous breaking of $\mathrm{B}-\mathrm{L}$ and $\mathrm{I}_{3 R}$ at a high scale $\mathrm{M}_{\mathrm{B}-\mathrm{L}}$, both already introduced in Ref. [6], the RH neutrinos can and generically will acquire a superheavy Majorana mass $\left(M\left(\nu_{R}\right) \sim M_{B-L}\right)$ violating B-L by two units. The $\nu_{L}$ and $\nu_{R}$ will of course combine to get a Dirac mass $\left(m(\nu)_{\text {Dirac }}\right)$ through electroweak symmetry breaking. The idea of the seesaw [37] is simply this. Ignoring family mixing for a moment, it combines the superheavy Majorana mass of $\nu_{R}$ with the Dirac mass (through diagonalization) to yield a mass for $\nu_{L}$ given by

$$
m\left(\nu_{L}\right) \approx m(\nu)_{\text {Dirac }}^{2} / M\left(\nu_{R}\right)
$$

which is naturally superlight if $M\left(\nu_{R}\right)$ is naturally superheavy.

\footnotetext{
${ }^{\mathrm{f}}$ Note if neutrinos were strictly massless, that feature could be understood simply by assuming the two-component theory of the neutrino (as in the SM or SU(5)) and lepton number conservation (despite quantum gravity). Such masslessness of the neutrinos, with this reasoning, is in fact what a large number of physicists believed until the 1980s and even late 1990s. Alternatively, if neutrinos had masses $\lesssim 10^{-5} \mathrm{eV}$ (assuming that could be detected), that too can be understood in the SM and $\mathrm{SU}(5)$ by allowing for quantum gravity-induced lepton number violation (see text). It is the superK scale $\sim 1 / 20 \mathrm{eV}$, that is neither zero nor as small as $10^{-5} \mathrm{eV}$, that cannot be accounted for naturally by either of these two alternatives. It thus calls for new physics beyond the SM.
} 
The seesaw mechanism in turn of course needs the ideas of SU(4)-color and SUSY unification so as to be quantitatively useful. This is because these two ideas serve to determine the Dirac and the Majorana masses (especially for the third family) rather well, which would otherwise be quite arbitrary. To be specific, $\mathrm{SU}(4)$-color treats $\nu_{R, L}^{\tau}$ as the fourth color partners of $t_{R, L}$. As a result, it yields (see Eq. 1(b)): $m\left(\nu^{\tau}\right)_{\text {Dirac }} \approx$ $m_{\text {top }}\left(M_{\mathrm{GUT}}\right) \approx 120 \mathrm{GeV}$. [Here, we are ignoring family-mixing; which, however, would introduce insignificant correction to this relation for hierarchical fermion massmatrices, see Section 4.]

Furthermore, by having B-L as a local symmetry, SU(4)-color naturally associates the Majorana mass of $\nu_{R}^{\tau}$ with the (B-L)-breaking scale, and thereby, for the sake of coupling unification, with the GUT-scale. In the context of a minimal Higgs system, which breaks $\mathrm{B}-\mathrm{L}$ by one unit, one thereby obtains (see next section): $M\left(\nu_{R}^{\tau}\right) \sim\left(M_{B-L}\right)^{2} / M \approx\left(M_{\mathrm{GUT}}^{2} / M\right) \approx\left(2 \times 10^{16} \mathrm{GeV}\right)^{2} /\left(10^{18} \mathrm{GeV}\right)(1 / 2-2) \approx$ $\left(4 \times 10^{14} \mathrm{GeV}\right)(1 / 2-2)$. Here $M_{B-L}$ denotes the VEV of the Higgs field that breaks B-L by one unit; for reasons mentioned above it is identified with the GUTscale. The mass $\mathrm{M}$ denotes the scale of an effective non-renormalizable operator induced by Planck or string-scale physics (see Section 4); we have therefore set $M \approx\left(10^{18} \mathrm{GeV}\right)(1 / 2-2)$. Substituting these values of $m\left(\nu^{\tau}\right)_{\text {Dirac }}$ and $M\left(\nu_{R}^{\tau}\right)$ into Eq. (41) and ignoring 2-3 family mixing for a moment (which turns out to be unimportant for mass eigenvalues), one thus obtains:

$$
\left.m\left(\nu_{L}^{3}\right) \approx(120 \mathrm{GeV})^{2} /\left(4 \times 10^{14} \mathrm{GeV}(1 / 2-2)\right) \approx(1 / 28 \mathrm{eV})(1 / 2-2)\right) .
$$

With hierarchical pattern for fermion mass-matrices (see Sec. 4), one necessarily obtains $m\left(\nu_{L}^{2}\right) \ll m\left(\nu_{L}^{3}\right)$ and thus $\sqrt{\Delta m_{23}^{2}} \approx m\left(\nu_{L}^{3}\right) \sim 1 / 28 \mathrm{eV}(1 / 2-2)$. This is just the right magnitude matching the mass scale observed at SuperK [24]!

Without an underlying reason as above for at least the approximate values of these two vastly differing mass-scales - $m\left(\nu_{\text {Dirac }}^{\tau}\right)$ and $M\left(\nu_{R}^{\tau}\right)$ - the seesaw mechanism by itself would have no clue, quantitatively, to the mass of the LH neutrino. In fact it would yield a rather arbitrary value for $m\left(\nu_{L}^{\tau}\right) \approx m\left(\nu_{L}^{3}\right)$, which could vary quite easily by more than 10 orders of magnitude either way around the observed mass scale (that is from about $10^{-14} \mathrm{eV}$ to nearly $10 \mathrm{GeV}$, say). This would in fact be true if one introduces the $\mathrm{RH}$ neutrinos as a singlet of the $\mathrm{SM}$ or of $\mathrm{SU}(5) .{ }^{\mathrm{g}}$ Within symmetries like $\mathrm{G}(2213)=\mathrm{SU}(2)_{L} \times \mathrm{SU}(2)_{R} \times \mathrm{U}(1)_{B-L} \times \mathrm{SU}(3)^{c}$ [29] and $[\mathrm{SU}(3)]^{3}$ [20], which

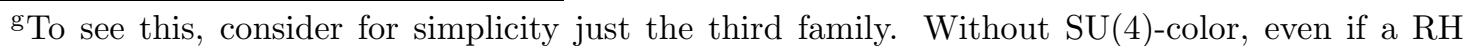
two-component fermion $N$ (the analogue of $\nu_{R}$ ) is introduced by hand as a singlet of the gauge symmetry of the SM or $S U(5)$, such an $N$ by no means should be regarded as a member of the third family, because it is not linked by a gauge transformation to the other fermions in the third family. Thus its Dirac mass term given by $m\left(\nu_{\text {Dirac }}^{\tau}\right)\left[\bar{\nu}_{L}^{\tau} N+\right.$ h.c. $]$ can vary from say $100 \mathrm{GeV}$ to even $1 \mathrm{MeV}$. Likewise, without $\mathrm{B}-\mathrm{L}$, the Majorana mass $M(N)$ can be as high as the Planck or the string scale $\left(10^{18}-10^{17} \mathrm{GeV}\right)$, and as low as say $1 \mathrm{TeV}$. This would yield $m\left(\nu_{L}^{\tau}\right)$ varying from about $10^{-14} \mathrm{eV}$ to about $10 \mathrm{GeV}$. Such arbitrariness both in the Dirac and in the Majorana masses, is drastically reduced, however, once $\nu_{R}$ is related to the other fermions in the family by an $S U(4)$-color gauge transformation and SUSY unification is assumed (see discussion preceding Eq. (5)). 
possess $\mathrm{B}-\mathrm{L}, \mathrm{RH}$ neutrino is a compelling feature; so the Majorana mass of $\nu_{R}^{\tau}$ can be constrained. But the Dirac mass of $\nu_{\tau}$ is not related by any symmetry to the top mass. It can thus vary from say $1 \mathrm{MeV}$ to $100 \mathrm{GeV}$. This would render the prediction of $\nu_{L}^{\tau}$-mass (within $\mathrm{G}\left(2213\right.$ ) or $[\mathrm{SU}(3)]^{3}$ ) uncertain by almost ten orders of magnitude for a given $M\left(\nu_{R}^{\tau}\right)$ (see Eq. (44) $)$. They also do not yield $m_{b}\left(M_{\mathrm{GUT}}\right) \approx m_{\tau}$ (Eq. 1(a)). Now, as mentioned before, flip $\mathrm{SU}(5) \times \mathrm{U}(1)$ possesses $\nu_{R}$ and $\mathrm{B}-\mathrm{L}$, and yields Eq. 1(b), but not Eq. 1(a).

In short, the seesaw mechanism needs (for a quantitative success) the ideas of SUSY unification and SU(4)-color, and of course vice-versa; only together they provide an understanding of neutrino masses as observed, explaining the large hierarchy such as $m\left(\nu_{L}^{\tau}\right) / m_{\text {top }} \sim 10^{-12}$ (Eq. (44) ). Schematically, one thus finds:

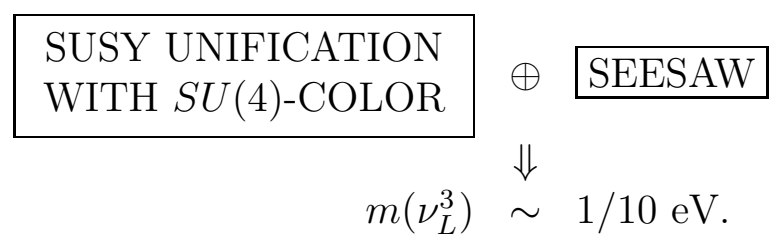

By the same token, as claimed in the introduction and in Section 2, the agreement of the theoretically expected $\sqrt{\Delta m^{2}(\nu)_{23}}$ with the observed SuperK value, together with the success of the mass-relations $1(\mathrm{a})$ and $1(\mathrm{~b})$, clearly seem to favor the idea of the seesaw and select out the route to higher unification based on SU(4)-color and supersymmetry, as opposed to other alternatives. This is further strengthened by the success of leptogenesis discussed in Section 6.

\section{Fermion Masses and Neutrino Oscillations}

I will now present the results of a specific attempt, by Babu, Pati and Wilczek 38, to understand some aspects of the masses and mixings of fermions including the neutrinos in the context of $\mathrm{SO}(10)$ or $\mathrm{G}(224)$ symmetry. For operational purposes, we assume, together with $\mathrm{SO}(10)$, a commuting $\mathrm{U}(1)$-flavor symmetry 30] which distinguishes between different families and leads to the desired gross hierarchical pattern in the mass-matrices (see below). Here again one sees how essentially the group theory of $\mathrm{SO}(10)$ or $\mathrm{G}(224)$ serves to explain, in the context of a minimal Higgs system (see below), some intriguing features such as the smallness of $V_{c b} \approx 0.04$ and the largeness of $\theta_{23}^{\nu} \approx \pi / 4$ in a compelling manner, together with $m_{b}(\mathrm{GUT}) \approx m_{\tau}$, and $m_{s}(\mathrm{GUT}) \neq m_{\mu}$. Owing to length limitation I will leave out much of the discussion

${ }^{\mathrm{h}}$ Although I have argued that the data suggests the need for SU(4)-color in 4D, I should mention a possible exception. If either $\mathrm{G}(2213)$ or $[\mathrm{SU}(3)]^{3}$ or flip $\mathrm{SU}(5) \times \mathrm{U}(1)$ emerges in $4 \mathrm{D}$ through a string solution and if the latter turns out to ensure SU(4)-color relations for the Yukawa couplings (especially both Eqs. 1(a) and 1(b)) near the string scale, then for all practical purposes the advantages of $\mathrm{SU}(4)$-color would persist in $4 \mathrm{D}$, since $\mathrm{B}-\mathrm{L}$ is already contained in the gauge symmetries mentioned above. 
in this regard, and would refer the reader to the original paper [38, and also to the subsequent review talks [30].

The BPW framework assumes that the fermion mass-matrix is generated through only a minimal Higgs system, which also serves to break $\mathrm{SO}(10)$ to $S U(3)^{c} \times U(1)_{\text {em }}$. It consists of the set: ${ }^{\mathrm{i}} H_{\text {minimal }}=\left\{\mathbf{4 5} \mathbf{\mathbf { H }}, \mathbf{1 6} \mathbf{\mathbf { H }}, \overline{\mathbf{1 6}}_{\mathbf{H}}, \mathbf{1 0} \mathbf{\mathbf { H }}, \mathbf{S}\right\}$, where $S$ is a singlet of $\mathrm{SO}(10)$ carrying appropriate $\mathrm{U}(1)_{F}$ flavor-charge [30. This Higgs system is regarded as minimal in the sense that it consists only of lowest dimensional multiplets $\mathrm{j}$ in contrast to large-dimensional tensorial multiplets (e.g. $126_{H}+\overline{126}_{H}+210_{H}+$ possibly $120_{H}+10_{H}$ ) which have also been used widely in the literature [42. The advantages of low-dimensional over the large-dimensional multiplets and vice verse are discussed elsewhere [30] (see, e.g. the third paper).

Of the Higgs multiplet shown above, the VEV of $\left\langle\mathbf{4} \mathbf{5}_{\mathbf{H}}\right\rangle \sim M_{U}$ breaks $S O(10)$ in the B-L direction to $G(2213)=S U(2)_{L} \times S U(2)_{R} \times U(1)_{B-L} \times S U(3)^{c}$, and those of $\left\langle\mathbf{1 6}_{\mathbf{H}}\right\rangle=\left\langle\overline{\mathbf{1 6}}_{\mathbf{H}}\right\rangle \sim M_{U}$ along $\left\langle\tilde{\bar{\nu}}_{R H}\right\rangle$ and $\left\langle\tilde{\nu}_{R H}\right\rangle$ break $G(2213)$ into the SM symmetry $G(213)$ at the unification-scale $M_{U}$. Now $G(213)$ breaks at the electroweak scale by the VEVs of $\left\langle\mathbf{1 0}_{\mathbf{H}}\right\rangle$ and of the EW doublet in $\left\langle\mathbf{1 6}_{\mathbf{H}}\right\rangle$ to $S U(3)^{c} \times U(1)_{\mathrm{em}}$. The singlet $S$ is also assumed to have a VEV $\sim M_{U}$. It should be noted that although the VEVs of $16_{H}$ and $\overline{16}_{H}$ break B-L by one unit and thereby break the familiar R-parity, the system still preserves a discrete matter parity $\left(16_{i} \rightarrow-16_{i}, 16_{H} \rightarrow 16_{H}, \overline{16}_{H} \rightarrow \overline{16}_{H}\right.$, $45_{H} \rightarrow 45_{H}, 10_{H} \rightarrow 10_{H}, S \rightarrow S$, gauge multiplet $\rightarrow$ itself). This suffices to ensure a stable LSP and absence of $\mathrm{d}=4$ proton decay operators.

With the minimal Higgs system, fermions receive Dirac masses through three types of allowed $\mathrm{SO}(10)$-invariant effective couplings: (i) $h_{i j} 16_{i} 16_{j} 10_{H}$; (ii) $a_{i j} 16_{i} 16_{j} 10_{H} 45_{H} / M$; and (iii) $g_{i j} 16_{i} 16_{j} 16_{H} 16_{H}^{d} / M$, where $(i, j)=1,2,3$ represent family indices. ${ }^{\mathrm{k}}$ Each of these coupling parameters like $h_{i j}, a_{i j}$ and $g_{i j}$ carry suitable powers of $(\langle S\rangle / M)$, depending upon flavor symmetry [30]. Such powers and/or higher dimensional operators like (ii) and (iii) induce the desired hierarchical couplings

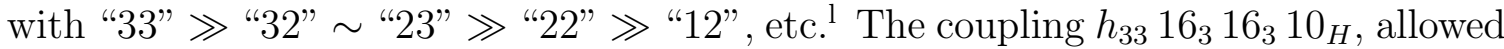
by $\mathrm{SO}(10)$ and $\mathrm{U}(1)_{F}$ flavor symmetry, is the leading term with $h_{33} \approx h_{\text {top }} \sim 1$, that gives masses to the third family. Higher dimensional operators like (ii) and (iii) are expected to arise through quantum gravity or string-scale physics so that $M \sim M_{\text {st }}$ or

\footnotetext{
${ }^{\mathrm{i}}$ Analogous multiplets are used for the case of $\mathrm{G}(224)$.

${ }^{j}$ Such lowest dimensional Higgs multiplets have also been considered by other authors 39, 40. with similar degree of success. For what it is worth, solutions of weakly interacting heterotic string theories do yield such low-dimensional multiplets, though not higher dimensional ones like 126 and 120 41.

${ }^{\mathrm{k}}$ Here $16_{H}^{d}$ denotes that the lepton-like doublet in $16_{H}$, having the quantum numbers of $\mathrm{H}_{d}$. It acquires a VEV of the electroweak scale due to its mixing with the down-type doublet in $10_{H}$. 38.

${ }^{1}$ As an example, the desired hierarchical pattern mentioned above can be achieved simply by assigning the $\mathrm{U}(1)_{F}$-charges as follows: $(\mathrm{a}, \mathrm{a}+1, \mathrm{a}+2,-2 \mathrm{a},-\mathrm{a}-1 / 2,-\mathrm{a}, 0,-1)$ to $\left(16_{3}, 16_{2}, 16_{1}, 10_{H}\right.$, $16_{H}, \overline{16}_{H}, 45_{H}, \mathrm{~S}$ ), with a $=1 / 2$. Origin of such a flavor symmetry must be sought in an underlying theory like string theory.
} 
$M_{\mathrm{pl}} \sim 10^{18} \mathrm{GeV}(1 / 2-2) .{ }^{\mathrm{m}}$ The coupling involving $\left\langle 10_{H}\right\rangle \cdot\left\langle 45_{H}\right\rangle / M$ provides (B-L)dependent family-antisymmetric coupling that contributes only to off-diagonal mixings, while $\left\langle 16_{H}\right\rangle \cdot\left\langle 16_{H}^{d}\right\rangle / M$ contributes only to the down flavor sector and thereby to CKM mixings 38 .

In addition to these couplings, which provide Dirac masses, the $\mathrm{RH}$ neutrinos receive hierarchical Majorana masses through effective couplings of the form $f_{i j} 16_{i} 16_{j}$ $\overline{16}_{H} \overline{16}_{H} / M$; where $f_{i j}$ 's contain appropriate powers of $\langle S\rangle / M$, dictated by flavor symmetry [30. The leading contribution arises again for the third family with $f_{33} \sim 1$, so that $M\left(\nu_{R}^{3}\right) \approx f_{33}\left\langle\overline{16}_{H}\right\rangle^{2} / M \approx\left(2 \times 10^{16} \mathrm{GeV}\right)^{2} /\left(10^{18} \mathrm{GeV}\right)(1 / 2-2) \approx 4 \times$ $10^{14} \mathrm{GeV}(1 / 2-2)$ (as noted in Section 3).

Assuming for simplicity $\mathrm{CP}$ conservation for a moment [38], so that all entries in the fermion mass-matrices are real, it turns out that the number of effective parameters ( 7 for the Dirac sector) are significantly less than the observables (12 for the quark and charged lepton sectors). We determine these parameters by using $m_{t}^{\text {phys }}=$ $174 \mathrm{GeV}, m_{c}\left(m_{c}\right)=1.37 \mathrm{GeV}, m_{s}(1 \mathrm{GeV})=110-116 \mathrm{MeV}, m_{u}(1 \mathrm{GeV})=6 \mathrm{MeV}$ and the masses of $e, \mu$ and $\tau$ as inputs. For the Majorana mass matrix of the RH neutrinos, $\mathrm{U}(1)$-flavor symmetry [30] fixes the "23" element $y$ to be of order $1 / 10$ relative to the "33" element, which is normalized to 1 in units of $M\left(\nu_{R}^{3}\right)$. It turns out 38] that with such a hierarchical $|y| \sim 1 / 10$, an input value of $m\left(\nu_{2}\right) / m\left(\nu_{3}\right) \approx 1 / 5-1 / 7$ (as suggested by the data) can be satisfied provided $y$ is negative ${ }^{\mathrm{n}}$ with a value $y \approx-1 / 17$. One is then led to the following seven predictions:

\footnotetext{
${ }^{\mathrm{m}}$ I should add that some authors have exhibited a strong preference for using only renormalizable Yukawa interactions (effective or not). Such a preference does not seem to be warranted, however, because effective (apparently) non-renormalizable interactions do routinely arise at low energies by utilizing purely renormalizable interactions at high energies. That is the case for example for the four fermion weak interaction, and also for the effective interactions leading to seesaw neutrino masses (Type I or Type II). In the present case, one knows that there exists physics above the GUT scale, characterized by the string or the Planck scale. Thus, if one ignores such effective interactions, which are allowed by all symmetries, and which would be relevant especially for the masses of the first two families, the question might arise: Why?

${ }^{\mathrm{n}}$ In turn, this negative sign for $y$ implies [38] that the contributions to $\theta_{23}^{\nu}$ from the charged lepton and neutrino sectors add to each other rather than subtract (see Eq. (6)). Equally important is the fact that the charged lepton contribution to $\theta_{23}^{\nu}$ gets enhanced relative to the familiar mixing angle of $\sqrt{m_{\mu} / m_{\tau}}$ by a (B-L)-dependent family-antisymmetric factor $(\eta-3 \epsilon)^{1 / 2} /(\eta+3 \epsilon)^{1 / 2}$, while the analog of this last factor provides a suppression for $V_{c b}$, just as needed! (See the references [38, 30] for details of this discussion.) The combination of these two ingredients (i.e. addition of the two contributions and (B-L)-dependent enhancement) ends up in yielding a nearly maximal $\theta_{23}^{\nu}$, while $V_{c b}$ is small.
} 


\section{Predictions}

$$
\begin{aligned}
m_{b}\left(m_{b}\right) & \approx(4.7-4.9) \mathrm{GeV} & \approx 4.2 \mathrm{GeV} \\
\sqrt{\Delta m^{2}(\nu)_{23}} & \approx(1 / 24 \mathrm{eV})(1 / 2-2) & \approx 1 / 20 \mathrm{eV} \\
V_{c b} & \approx 0.044 & \approx 0.042 \\
\theta_{23}^{\nu} & \approx\left|(0.437)_{\text {ch.lep. }}+\sqrt{m\left(\nu_{2}\right) / m\left(\nu_{3}\right)}\right| & \\
\sin ^{2} 2 \theta_{23}^{\nu} & \approx 0.97-0.995\left(\text { for } \frac{m\left(\nu_{2}\right)}{m\left(\nu_{3}\right)} \approx \frac{1}{7}-\frac{1}{5}\right) & \approx 0.90-1 \\
V_{u s} & \approx 0.20 & \approx 0.225 \\
V_{u b} & \approx 0.0031 & \approx 0.0039 \\
m_{d}(1 \mathrm{GeV}) & \approx 8 \mathrm{MeV} & \approx 5-8 \mathrm{MeV} .
\end{aligned}
$$

Leaving aside $\left|V_{u b}\right|$, which is lower than the observed value by about $20 \%,{ }^{\circ}$ it is indeed remarkable, considering the bizarre pattern of fermion masses and mixings, that all the remaining six predictions agree with the data to within about $10 \%$ ! Particularly intriguing are the (B-L)-dependent enhancement for $\theta_{23}^{\nu}$ versus suppression for $V_{c b}$, as well as the fact that the contributions from the charged lepton and neutrino sectors necessarily add [38] in the case of $\theta_{23}^{\nu}$ (for a hierarchical $y$ ) (see Eq. (7)) but the corresponding terms for $V_{c b}$ subtract. It is the combination of these two factors which end up in yielding a nearly maximal $\theta_{23}^{\nu}$, and simultaneously a small $V_{c b}$, as needed (see footnote $m$ and references [38, 30]). It should be stressed that this explanation of the largeness of $\theta_{23}^{\nu}$ is characteristically different from all the other explanations in the literature. (Contrast for example from the lop-sided $\mathrm{SO}(10)$-models 39, where the largeness of $\theta_{23}^{\nu}$ arises entirely from the charged lepton sector.) It should also be noted that although $m\left(\nu_{2}\right) / m\left(\nu_{3}\right)$ varies with $y$, the prediction of $\theta_{23}^{\nu}$ is fairly stable as long as $|y|$ has a hierarchical value $(\sim 1 / 10$, within a factor of 3 , say), as suggested by flavor symmetry [30. In this sense, the near maximality of $\theta_{23}^{\nu}$ is a compelling prediction of the model.

It has been noted [30] that small non-seesaw contribution arising from a higher dimensional operator, consistent with flavor symmetry and $\mathrm{SO}(10)$, can contribute to the $\nu_{L}^{e} \nu_{L}^{\mu}$ mass term $\sim$ few $\times 10^{-3} \mathrm{eV}$; this can quite plausibly lead to large $\nu_{e}-\nu_{\mu}$ oscillation angle in accord with the LMA MSW solution to the solar neutrino puzzle: Including this we get:

$$
\begin{aligned}
& m\left(\nu_{2}\right) \approx(9-6) \times 10^{-3} \mathrm{eV} \text { (from seesaw) } \\
& m\left(\nu_{1}\right) \approx(1-\text { few }) \times 10^{-3} \mathrm{eV} ; \text { thus } \Delta \mathrm{m}_{12}^{2} \approx(4-8) \times 10^{-5} \mathrm{eV}^{2} \\
& \sin ^{2} 2 \theta_{12}^{\nu} \approx(0.5-0.7) \quad(\text { from non seesaw) } \\
& \theta_{13} \lesssim(2-5) \times 10^{-2} .
\end{aligned}
$$

${ }^{\circ}$ Such $20 \%$ discrepancy in $\left|V_{u b}\right|$ gets corrected without affecting other entries when one includes $\mathrm{CP}$ violation (see next section). 
Here, $m\left(\nu_{2}\right) / m\left(\nu_{3}\right)$ and thus $m\left(\nu_{2}\right)$ is an input that fixes the choice of $y$ (see above). It should be mentioned that in contrast to the near maximality of $\theta_{23}^{\nu}$, which emerges as a compelling prediction, largeness of $\theta_{12}^{\nu}$ is a plausible possibility, but not a prediction of the framework. The superheavy Majorana masses of the $\mathrm{RH}$ neutrinos $\nu_{R}^{i}$ also get fairly fixed within the model by the flavor-hierarchy, one gets [38, 30]:

$$
\left(M_{3}, M_{2}, M_{1}\right) \approx\left(4 \times 10^{14}(1 / 2-2) ; 10^{12}(1 / 2-2) ; 4 \times 10^{10}(1 / 8-4)\right) \mathrm{GeV} .
$$

Note that both the light and the superheavy neutrino masses have a normal hierarchy: $m_{1} \lesssim m_{2} \ll m_{3}$ and $M_{1} \ll M_{2} \ll M_{3}$. The latter hierarchy would be important for leptogenesis.

\section{$5 \quad \mathrm{CP}$ and Flavor Violations}

To incorporate $\mathrm{CP}$ violations into the $\mathrm{G}(224) / \mathrm{SO}(10)$-framework outlined in the previous section [38, the simplest and most attractive possibility appears to be to assume that $\mathrm{CP}$ violation arises spontaneously, either entirely or dominantly, through the VEVs of some or all of the Higgs fields (in our case $45_{H}, 16_{H}, \overline{16}_{H}, 10_{H}$ and/or $S$ ), and thus through phases in the fermion mass matrices 43 . These latter would induce $\mathrm{CP}$ violation both through SM CKM-interactions as well as through supersymmetric interactions involving sfermion-gaugino loops.

Motivated by the feebleness of flavor changing neutral current interactions, we assume that SUSY-breaking is flavor-universal and CP-conserving at a high scale $\left(M^{*} \gtrsim M_{\mathrm{GUT}}\right)$. SUSY contributions still violate $\mathrm{CP}$ and flavor owing to renormalization group-evolution of the scalar masses and the A-parameters from the high messenger scale $M^{*}$ to low scales, involving Yukawa couplings which are flavordependent [44, and because of phases in the fermion mass matrices.

The challenge then is this: Can the idea outlined above be implemented by including both the SO(10)-based SM and the SUSY contributions so that the framework would be consistent with the observed $\mathrm{CP}$ and/or flavor violations - as in $\Delta m_{K}, \epsilon_{K}$, $\Delta m_{B_{d}}$ and $S\left(B_{d} \rightarrow J / \psi K_{S}\right)$ - while still preserving its successes [38] listed in the previous section as regards the predictions of the fermion masses and neutrino oscillations (see Eq. (6))? Furthermore, does the SUSY G(224)/SO(10)-framework with $\mathrm{CP}$ violation introduced as above [43] lead to some characteristic predictions that can help distinguish it from the SM and other alternative frameworks?

The first question raised above poses a non-trivial challenge especially because all four CP and/or flavor violating entities listed above agree rather well with the predictions of the standard CKM model for a single choice of the Wolfenstein parameters (see e.g. Ref. 45]):

$$
\bar{\rho}_{W}=0.204 \pm 0.035: \quad \bar{\eta}_{W}=0.336 \pm 0.021(1 \sigma) .
$$

The questions raised above are addressed in three recent papers by Babu, Parul Rastogi and myself [43, 46, 47]. It turns out that the answers to both questions are 
in the affirmative. True distinctions of the SUSY G(224)/SO(10) framework [38] outlined above from the $\mathrm{SM}$ as well as alternative $\mathrm{SO}(10)$-frameworks (e.g. of AlbrightBarr [39]) arise though its predictions, for example, for the edm's of the neutron and the electron and the rates for $\mu \rightarrow e \gamma$ and $\tau \rightarrow \mu \gamma$. I will leave out the discussions in this regard and mention only the essence of the results. I would refer the reader to the original papers [43, 46, 47] for details of clarification. The results are as follows:

1. Allowing for phases $(\sim 1 / 10$ to $1 / 2)$ in the parameters of the $\mathrm{G}(224) / \mathrm{SO}(10)$ based fermion mass matrices [38, it is found that there do exist solutions which yield masses and magnitudes of mixings of quarks and leptons including neutrinos, all in good accord with observations (to within 10\%), and at the same time yield the following values for the Wolfenstein parameters:

$$
\left(\bar{\rho}_{W}\right)_{S O(10)} \approx 0.14-0.17 \text { and }\left(\bar{\eta}_{W}\right)_{S O(10)} \approx 0.35-0.38
$$

Note that these are fairly close to the SM-based phenomenological values (Eq. (10)). This, together with having the right magnitudes of the CKM mixings, is the reason why the SUSY G(224)/SO(10) model as developed in Refs. 38, 43, succeeds in accounting for the observed $\mathrm{CP}$ and flavor violation in the quark sector (see below). ${ }^{p}$

2. CP and Flavor Violations in Quark Sector. Including both the SO(10)based SM-contribution (and thus using Eq. (11)) and the SUSY-contribution ${ }^{q}$ (with a plausible choice of the SUSY-spectrum-e.g. corresponding to $m_{o} \sim$ (550-650) $\mathrm{GeV}$ and $\left.m_{1 / 2} \sim(250-300) \mathrm{GeV}\right), m_{s q} \approx(0.8-1) \mathrm{TeV}, m_{\tilde{\ell}} \sim 600$ $\mathrm{GeV}, m_{\tilde{g}} \sim 120 \mathrm{GeV}$, and $x=m_{\tilde{g}} / m_{s q}^{2} \approx 0.6-0.8, M^{*} / M_{\mathrm{GUT}} \approx 3$ we obtain [43]:

$$
\begin{aligned}
& \left(\Delta m_{K}\right)_{\text {short dist }} \approx 3 \times 10^{-15} \mathrm{GeV} ; \quad \epsilon_{K} \approx(2-2.5) \times 10^{-3} \\
& \left(\Delta m_{B_{d}}\right) \approx(3.5-3.6) \times 10^{-13} \mathrm{GeV} ; \quad S\left(B_{d} \rightarrow J / \psi K_{s}\right) \approx 0.68-0.74 \\
& S\left(B_{d} \rightarrow \phi K_{s}\right) \approx 0.65-0.73 \\
& \Delta m_{B_{s}} \approx 17.3 \mathrm{ps}^{-1} ; \quad S\left(B_{s} \rightarrow J / \psi \phi\right) \approx \text { few } \% \\
& A(b \rightarrow s \gamma)_{S U S Y}^{S O(10)} \approx(1-5) \% \text { of } A(b \rightarrow s \gamma)_{S M} \\
& (e d m)_{\text {Aind }}^{\text {neutron }} \approx(1.6,1.08) \times 10^{-26} \mathrm{ecm}(\tan \beta=5,10) \\
& (e d m)_{\text {Aind }}^{\text {electron }} \approx\left(1.1 \times 10^{-28} / \tan \beta\right) e c m .
\end{aligned}
$$

${ }^{\mathrm{p}}$ Note that a priori a given $\mathrm{SO}(10)$ model with a certain pattern of fermion mass matrices need not yield $\left(\bar{\rho}_{W}, \bar{\eta}_{W}\right)$ lying even in the first quadrant (not to mention having the right magnitudes), for any choice of phases and magnitudes of the parameters of the mass-matrices, without conflicting with the observed fermion masses and mixings (see e.g. Ref. [48]).

${ }^{\mathrm{q} I}$ should stress that for a given choice of the few SUSY parameters (i.e. $m_{0}, M_{1 / 2}$ and $M^{*}$ ), SUSY CP and flavor violations (both the magnitudes and the phases) are completely determined in the model owing to prior works $[38,43$, that successfully describes fermion masses and neutrino oscillations and yields $\left(\bar{\rho}_{W}, \eta \bar{W}\right)$ as in Eq. (11). 
Here, we have used central values of the lattice results on the matrix element

$\widehat{B}_{k}=0.86, f_{B_{d}} \sqrt{\widehat{B}_{B_{d}}}=215 \mathrm{MeV}$, and $f_{B_{s}} \sqrt{\widehat{B}_{B_{s}}}=245 \mathrm{MeV}$. Note that the first four entities $\left(\Delta m_{K}, \epsilon_{K}, \Delta m_{B_{d}}\right.$ and $\left.S\left(B_{d} \rightarrow J / \psi K_{s}\right)\right)$, which are well measured, are all in good agreement with observations (within 10\%). In all these cases, the SUSY-contribution turns out to be rather small ( $\leq 5 \%$ in amplitude) compared to the SM-contribution, except however for $\epsilon_{K}$, for which it is sizeable $(\approx 20-30 \%)$ and relatively negative. This negative contribution to $\epsilon_{K}$ can help distinguish the SUSY G(224)/SO(10) model from the SM, once the matrix element $\widehat{B}_{K}$ is determined to $5 \%$ accuracy. Given the present experimental limit on $(\mathrm{edm})_{n}<6.3 \times 10^{-26} \mathrm{ecm}$, the predicted value of $(1.6-1) \times 10^{-26} \mathrm{ecm}$ is in an extremely interesting range and can be probed with an improvement in the current limit by a factor of 10 . The predicted value of $S\left(B_{d} \rightarrow \phi K_{s}\right)$, which is close to the SM-value but is at present about $2 \sigma$ away from the BaBar-BELLE average value of $(0.47 \pm 0.19)$, would also provide a very interesting test once it is better measured, and so also would the parameters of the $B_{s}$ system, which are predicted to nearly coincide with the predictions of the SM.

As a summary of this section, we see that the SUSY G(224) or SO(10)-framework (remarkably enough) has met all the challenges so far in being able to reproduce the observed features of CP and quark-flavor violations, as well as of fermion masses and neutrino oscillations; and it has predictions than can probe the framework further!

\section{Lepton Flavor Violation}

Lepton flavor violating processes (such as $\mu \rightarrow e \gamma, \tau \rightarrow \mu \gamma, \mu N \rightarrow e N$, etc.) can provide sensitive probes into SUSY grand unification. In our case, these get contributions from three sources [46]: (i) Slepton (mass) ${ }^{2}$-elements $\left(\delta m^{2}\right)_{L L}^{i j}$ arising from RG-running of scalar masses from $M^{*}$ to $M_{\mathrm{GUT}}\left(M^{*}\right.$ being the messenger scale where SUSY-breaking parameters are flavor-universal); (ii) $\left(\delta m^{2}\right)_{L R}^{i j}$ which arise from Aterms induced through RG-running from $M^{*}$ to $M_{\mathrm{GUT}}$; and (iii) $\left(\delta m^{2}\right)_{L L}^{i j}$ arising from RG-running of scalar masses from $M_{\mathrm{GUT}}$ to the RH neutrino masses $M_{R i}$. The first two effects, though important as long as $\ell n\left(M^{*} / M_{\mathrm{GUT}}\right) \gtrsim 1$, are invariably dropped in the literature. For a given choice of the SUSY spectrum (i.e. $m_{0}$ and $\left.m_{1 / 2}\right)$ and $M^{*}$, the predictions of the model are completely fixed in terms of the mass-matrices of the quarks, leptons and neutrinos, which as we saw are fixed following discussions in Sections 4 and 5 [38, 43].

The predictions for the branching ratios for the $\mathrm{SO}(10)$-model (for a sample choice of $\left(m_{0}, m_{1 / 2}\right)$, with $\ell n M^{*} / M_{\mathrm{GUT}}=1$; i.e. $\left.M^{*} \approx 2.7 M_{\mathrm{GUT}}\right)$ are as follows [46]:

$$
\begin{array}{c|c|c|c|c}
\left(m_{0}, m_{1 / 2}\right) & (600,300) & (450,300) & (100,440) & (400,300) \\
B(\mu \rightarrow e \gamma) & (3.3,9.8) \times 10^{-12} & (2.7,4.6) \times 10^{-11} & (1,1) \times 10^{-8} & (0.95,3.8) \times 10^{-11} \\
B(\tau \rightarrow \mu \gamma) & (2.4,3.1) \times 10^{-9} & (2.7,5.6) \times 10^{-9} & (8.3,8.4) \times 10^{-8} & (1.4,1.8) \times 10^{-8}
\end{array}
$$


Here, $\left(m_{0}, m_{1 / 2}\right)$ are given in $\mathrm{GeV}$. The two entries appearing under each column correspond to $\mu>0$ and $\mu<0$ respectively. The first three columns are for $\tan \beta=10$ and the last one for $\tan \beta=20$. The predictions for $B(\mu \rightarrow e \gamma)$ for the G(224)-model are lower by a factor $\approx$ 4-6 than those for the $S O(10)$-model ${ }^{\mathrm{r}}$ (see Ref. [43, 46]). Given the present empirical limit of $B(\mu \rightarrow e \gamma) \leq 1.2 \times 10^{-11}$ [49], we see that the case of $\left(m_{0}, m_{1 / 2}\right)=(100,440) \mathrm{GeV}$, is excluded for the $\mathrm{SO}(10)$ as well as for the $\mathrm{G}(224)$ model, while $\left(m_{0}, m_{1 / 2}\right)=(450,300) \mathrm{GeV}$ is excluded for the former though not for the latter, with $\tan \beta=10$. The interesting point is that, even if sleptons are rather heavy $\left(\left(m_{0}, m_{1 / 2}\right) \approx(800,250) \mathrm{GeV}\right.$, say), for which one finds [46] $(B(\mu \rightarrow e \gamma))_{S O(10)} \approx(2.9,17) \times 10^{-13}$ for $(\mu>0, \mu<0), \mu \rightarrow$ e $\gamma$ should be discovered with an improvement in the current limit by a factor of 10-100. This is being planned at the MEG experiment at PSI. The present empirical limit of $B(\tau \rightarrow \mu \gamma)<7 \times 10^{-8}$ obtained at BaBar [50] also excludes the choice $\left(m_{0}, m_{1 / 2}\right)=(100,440)$ Gev for the case of $\mathrm{SO}(10)$ with $\tan \beta=10$. One finds [46], however, that even if sleptons are rather heavy $(\lesssim 800 \mathrm{GeV}$, say), $\tau \rightarrow \mu \gamma$ should be discovered with improvement in the current limit by a factor of $10-50$.

Thus, we see that studies of lepton flavor violation can provide stringent tests of the SUSY SO(10)/G(224)-framework. They can even distinguish between SO(10) and G(224) symmetries. Although, I have not explicitly discussed, it turns out that they can also clearly distinguish between (for example) the hierarchical 38] versus lop-sided [39] SO(10)-models (see Ref. 47]).

\section{Baryogenesis Via Leptogenesis}

The observed matter-antimatter asymmetry is an important clue to physics at truly short distances. Given the existence of the RH neutrinos [6], which naturally can possess superheavy Majorana masses, violating lepton number by two units, baryogenesis via leptogenesis [27] has emerged as perhaps the most viable mechanism for generating the observed baryon asymmetry of the universe. The intriguing feature is that it relates our understanding of the neutrino masses to our own origin.

The question of whether this mechanism can quantitatively explain the observed baryon asymmetry depends however crucially on the Dirac as well as the Majorana mass-matrices of the neutrinos, including the phases therein and the eigenvalues of the Majorana matrix $\left(M_{1}, M_{2}\right.$ and $\left.M_{3}\right)$. This question has been considered in a recent work [28] based on a realistic $\mathrm{G}(224) / \mathrm{SO}(10)$-framework [38, 43] for fermion masses, neutrino oscillations and CP violation, as described in Sections 4 and 5. The advantage in this case is that the Dirac and the Majorana mass matrices including the phases in the Dirac sector are already determined by prior considerations [38, 43].

${ }^{\mathrm{r}}$ This is because the RG running of the scalar masses and the A-parameters from $M^{*}$ to $M_{\mathrm{GUT}}$ leads to larger effects for the case of $\mathrm{SO}(10)$ than for $\mathrm{G}(224)$. For distinguishing between these models, one would need to determine $\tan \beta$. 
This work has also been reviewed in recent talks [30. Here, I will present only the results and refer the reader to the references [28, 30] for details.

The basic picture is this. Within an inflationary scenario, the lightest RH neutrinos $\left(N_{1}\right.$ 's) with a mass $\approx 10^{10} \mathrm{GeV}\left(\frac{1}{3}-3\right)$ are produced either from the thermal bath following reheating $\left(T_{R H} \approx\right.$ few $\left.\times 10^{9} \mathrm{GeV}\right)$, or non-thermally from the decay of the inflaton (with $T_{R H}$ in this case being about $10^{7} \mathrm{GeV}$ ). In either case, the RH neutrinos $\left(N_{1}\right.$ 's) having Majorana masses decay by utilizing their Dirac couplings into both $\ell+H$ and $\bar{\ell}+\bar{H}$ (and corresponding SUSY modes), thus violating $\mathrm{B}-\mathrm{L}$. In the presence of $\mathrm{CP}$ violating phases, these decays produce a net lepton-asymmetry $Y_{L}=\left(n_{L}-n_{\bar{L}}\right) / s$ which is converted to a baryon asymmetry $Y_{B}=\left(n_{B}-n_{\bar{B}}\right) / s=C Y_{L}\left(C \approx-\frac{1}{3}\right.$ for MSSM $)$ by the electroweak sphaleron effects. For the thermal case, using $\left(M_{1} / M_{2}\right) \approx 4 \times 10^{-3}$ (see Eq. (9)), one obtains [28]:

$$
\left(Y_{B}\right)_{\text {Thermal }} / \sin 2 \phi_{\mathrm{eff}} \approx(10-30) \times 10^{-11}
$$

Here $\phi_{\text {eff }}$ denotes an effective phase depending upon phases in the Dirac as well as Majorana mass-matrices of the neutrinos. For the non-thermal case, using an effective superpotential so as to implement hybrid inflation [51] involving the GUT-scale VEVs of $(1,2,4)_{H}$ and $(1,2, \overline{4})_{H}$, and $M_{1} \approx\left(2 \times 10^{10} \mathrm{GeV}\right)(1-1 / 3)$ in accord with Eq. (9), one obtains 28 ]

$$
\left(Y_{B}\right)_{\text {Non-thermal }} / \sin 2 \phi_{\text {eff }} \approx(20-200) \times 10^{-11}
$$

with a reheat temperature $\approx(3-1) \times 10^{7} \mathrm{GeV}$, in accord with the gravitino-constraint. We see that the derived values of $Y_{B}$ can in fact account for the recently observed value $\left(Y_{B}\right)_{\mathrm{WMAP}} \approx(8.7 \pm 0.4) \times 10^{-11}$, for very natural values of the phase anglethat is $\sin 2 \phi_{\text {eff }} \approx(1 / 3-1)$ for the thermal case and $\sin 2 \phi_{\text {eff }} \approx(1 / 2-1 / 20)$, for the non-thermal case.

In summary, we see that the SUSY G(224)/SO(10)-framework [38, 43] described in Sections 4 and 5 provides a simple and unified description of not only fermion masses, neutrino oscillations, and $\mathrm{CP}$ violation, but also of baryogenesis via leptogenesis. In here, we see a beautiful link between our understanding of the light neutrino masses (using seesaw, SU(4)-color and SUSY unification) and our own origin!

\section{Proton Decay}

Proton Decay is perhaps the most dramatic prediction of grand unification possessing quark-lepton unification [5]- 7]. I have discussed proton decay in the context of the SUSY SO(10)/G(224)-framework, as presented here, in some detail in recent reviews [30, which are updates of the results of Ref. 38] and other works. Here I will present only a summary of the results.

In SUSY unification there are in general three distinct mechanisms for proton decay: (i) The familiar $\mathbf{d}=\mathbf{6}$ operators mediated by $X$ and $Y$-type gauge bosons 
of $\mathrm{SU}(5)$ and $\mathrm{SO}(10)$, which lead to $e^{+} \pi^{0}$ as the dominant mode with a lifetime $\sim 10^{35 \pm 1}$ years; (ii) the "standard" $\mathbf{d}=\mathbf{5}$ operators [52, which arise through the exchange of color-triplet Higgsinos which are in $5_{H}+\overline{5}_{H}$ of $\mathrm{SU}(5)$ or $10_{H}$ of $\mathrm{SO}(10)$; these lead to $\bar{\nu} K^{+}$and $\bar{\nu} \pi^{+}$as the dominant modes with lifetimes varying from about $10^{29}$ to $10^{34}$ years; and (iii) the "new" neutrino mass-related $\mathbf{d}=\mathbf{5}$ operators which can generically arise through the exchange of color-triplet Higgsinos in the $\left(16_{H}+\overline{16}_{H}\right)$ of $\mathrm{SO}(10)$ [38, 53; these also lead to $\bar{\nu} K^{+}$and $\bar{\nu} \pi^{+}$as the dominant modes with lifetimes quite plausibly in the range of $10^{32}-10^{34}$ years. One important feature of these "new" $d=5$ operators is that they can contribute to proton decay for either SO(10) or an effective G(224)-symmetry, and for the latter, they are the only source of proton decay.

Guided by recent calculation based on quenched lattice QCD in the continuum limit [54] and that of renormalization factors $A_{L}$ and $A_{S}$ for $\mathrm{d}=5$ [55], we take (see Ref. [30] for details): $\left|\beta_{H}\right| \approx\left|\alpha_{H}\right| \approx\left(0.009 \mathrm{GeV}^{3}\right)(1 / \sqrt{2}-\sqrt{2}) ; m_{\tilde{q}} \sim m_{\tilde{\ell}} \sim 1.2 \mathrm{TeV}$ $(1 / 2-2) ; m_{\widetilde{W}} / m_{\tilde{q}} \simeq 1 / 6(1 / 2-2) ; A_{L} \approx 0.32, A_{S} \approx 0.93 ; \tan \beta \approx 3 ; M_{X} \approx M_{Y} \approx$ $10^{16} \mathrm{GeV}(1 \pm 25 \%)$; and $A_{R}\left(d=6, e^{+} \pi^{0}\right) \approx 3.4$. Updating the detailed analysis of Ref. [38, the predicted rates of proton decay, for the SUSY SO(10)/G(224)framework [38, with the parameters as given above, have been presented in some detail in my review talks 30]. (Analogous studies have also been carried out by the authors of Ref. [56] and also by other authors; see Ref. [30].)

As a summary, with the inclusion of the standard as well as the "new" neutrinomass related $d=5$ operators, one obtains as a conservative upper limit: [30, 38.

$$
\Gamma_{\text {proton }}^{-1}(d=5) \leq\left(\frac{1}{3}-2\right) \times 10^{34} \text { years }\left(\begin{array}{c}
\mathrm{SUSY} \\
\mathrm{SO}(10) / \mathrm{G}(224)
\end{array}\right)
$$

with $\bar{\nu} K^{+}$and $\bar{\nu} \pi^{+}$as the dominant modes, and quite possibly $\mu^{+} K^{0}$ being prominent. For the $\mathrm{d}=6, e^{+} \pi^{0}$-mode, with parameters as mentioned above, one obtains:

$$
\Gamma_{d=6}^{-1}\left(p \rightarrow e^{+} \pi^{0}\right)_{\text {Theory }} \approx 10^{35 \pm 1} \text { years }
$$

These should be compared with the experimental limits set by superK studies [57]:

$$
\begin{aligned}
\Gamma^{-1}\left(p \rightarrow e^{+} \pi^{0}\right)_{\operatorname{expt}} & \geq 6 \times 10^{33} \text { years } \\
\Gamma^{-1}\left(p \rightarrow \bar{\nu} K^{+}\right)_{\operatorname{expt}} & \geq 2 \times 10^{33} \text { years } .
\end{aligned}
$$

The implications of the theoretical predictions vis a vis the present experimental limits on a next-generation detector are noted in the next section.

\section{A Summary}

In this talk, I have argued on empirical grounds that the evidence in favor of SUSY grand unification possessing the symmetry $\mathrm{SU}(4)$-color in $4 \mathrm{D}$ is rather strong. It includes: 
- Quantum numbers of the members of a family,

- Quantization of electric charge,

- $Q_{e^{-}} / Q_{p}=-1$,

- Gauge coupling unification,

- $m_{b}^{0} \approx m_{\tau}^{0}$

- $\sqrt{\Delta m^{2}(\nu)_{23}} \approx 1 / 20 \mathrm{eV}$,

- A maximal $\theta_{23}^{\nu} \approx \pi / 4$ with a minimal $V_{c b} \approx 0.04$, and

- Baryon Excess $Y_{B} \sim 10^{-10}$.

All of these features and more including (even) $\mathrm{CP}$ and flavor violations hang together neatly within a single unified framework based on a presumed string-derived $\mathrm{G}(224)$ or $\mathrm{SO}(10)$-symmetry in $4 \mathrm{D}$, with low-energy supersymmetry. It is hard to imagine that the neat fitting of all these pieces can be a mere coincidence. It thus seems pressing that dedicated searches be made for the two main missing pieces of this picture - that is supersymmetry and proton decay. The search for supersymmetry at the LHC and a possible future ILC is eagerly awaited. That for proton decay would need an improvement in the current sensitivity by about a factor ten (see previous section). That in turn will need a megaton-size underground detector which could also probe deeper into neutrino-physics as well as supernova physics. The need for such a detector can thus hardly be overstated.

\section{Concluding Remarks: A Wish and a Goal}

Before ending this talk I would like to say a few words on a point of view that I have alluded to in the introduction and have implicitly adopted throughout in the rest of the talk. This has to do with the presumed origin of a desired effective theory in 4D near the GUT/Planck scale from an underlying theory.

I have argued on empirical grounds that there is a need for having an effective grand unification-like symmetry possessing $\mathrm{G}(224)$ or $\mathrm{SO}(10)$-symmetry in 4D. Clearly such a symmetry aids much to our understanding as evidenced by the list in the preceding section. It, however, leaves much to be explained by a deeper theory. Needless to say, the origin of the three families, their hierarchical masses and mixings, the values of certain parameters (such as $\alpha_{e m}, \alpha_{s t}, G_{F}, m_{e}, m_{e} / m_{p}, m_{n}-m_{p}$ and $G_{N}$ ), the fact that we live in four dimensions, and most of all the utterly minuscule magnitude of the cosmological constant (dark energy), many of which appear to be "chosen" somehow so as to satisfy anthropic constraints, are among the deepest puzzles confronting us today.

I have also implicitly assumed the existence of an underlying unified theory including gravity - be it string/M theory or something yet unknown - that would describe nature in a predictive manner and explain some of its presently unexplainable features, of the type mentioned above. Such a theory inevitably would operate at very short distances $\left(\sim M_{\text {Planck }}^{-1}\right)$ and very likely in higher dimensions. It then becomes imperative, for reasons stated above, that such a theory, as and when it evolves to 
be predictive, should lead to an effective grand unification-like symmetry (possessing $\mathrm{SU}(4)$-color) in $4 \mathrm{D}$ near the string/GUT-scale, rather than the SM symmetry. If such a symmetry does emerge from the underlying theory as a preferred solution in $4 \mathrm{D}$, together with the other desired properties mentioned above such as the presence of three families with the (desired) hierarchical Yukawa couplings, it would serve as a very useful bridge between the underlying (string) theory and phenomenology. It would explain observations in the real world, beyond those encompassed by grand unification. Needless to say, if such a solution would also explain the observed cosmological constant (dark energy), one would be at a peak in the path of understanding.

The picture depicted above is of course clearly $a$ wish and a goal, yet to be realized. Entertaining such a wish amounts to hoping that the current difficulties of string/M theory as regards the large multiplicity of string vacua [58] and lack of selectivity (mentioned in the introduction) would eventually be overcome possibly through a better understanding and/or formulation of the theory, and most likely through the introduction of some radically new ingredients, ${ }^{\mathrm{s}}$

Entertaining such a hope no doubt runs counter to the recently evolved view of landscape [59], combined with anthropism [60]. Such a hope is nevertheless inspired, as mentioned in the introduction, by the striking successes we have had over the last 400 years in our attempts at an understanding of nature at a fundamental level. To mention only a few that occurred in the last 100 years, they include first and foremost the insights provided by the two theories of relativity and quantum mechanics. In the present context they include also the successes of the ideas of the standard model, grand unification and inflation. Each of these have aided in varying degrees to our understanding of nature. At the same time, interestingly enough, each of these has provided certain ingredients that are crucial to an understanding of the evolution of life, in accord with anthropic constraints.

Here, I have in mind, for example: (i) The special theory of relativity providing the equation $E=m c^{2}$, which is crucial to an understanding of energy-generation in stars; (ii) The radically new laws of quantum mechanics, which are crucial to understanding the stability of the atoms; (iii) The co-existence of quarks and leptons, together especially with the charge-ratio $\left(Q_{e^{-}}+Q_{p}\right) / Q_{p} \lesssim 10^{-19}$, that are crucial to the formation of atoms and equally important to the "exact" neutrality of atoms and thereby of the sun and the earth; (iv) The co-existence of the three gauge forcesweak, electromagnetic and strong - each of which plays a role in energy generation in the stars; note that both (iii) and (iv) are neatly explained within grand unification, subject to a testable assumption on symmetry breaking; (v) The existence of neutrinos having (a) zero electric charge and (b) non-vanishing but truly tiny masses $<1 \mathrm{eV}$ (with $m\left(\nu_{3}\right) / m_{\text {top }} \sim 10^{-12}$ ), as opposed to masses $\gg 50 \mathrm{eV}$ (say); both of these features are crucial to many stages of the cosmic drama including suitable structure formation and energy generation in stars; and as we saw in Section 7 the tiny neutrino

sPerhaps as radical as Bohr's quantization rule that selected out a discrete set of orbits from an unstable continuum, which in turn found its proper interpretation within quantum mechanics. 
masses may well be linked to the generation of baryon excess $Y_{B} \sim 10^{-10}$ in the universe; all of these features involving neutrinos, which certainly are in accord with anthropic reasoning, are neatly explained within the $\mathrm{G}(224)$ or $\mathrm{SO}(10)$-unification (see Sections 4 and 7); (vi) The gross homogeneity and isotropy of the universe, together with small density fluctuations, and simultaneously the density parameter $\Omega \equiv \rho / \rho_{c}$ being so incredibly close to the critical value of unity especially at early times $\left(i . e . \Omega_{\text {now }}^{\text {obs }}=\mathcal{O}(1)\right.$ means $\left.\left|\Omega\left(t_{E W} \sim 10^{-11} s\right)-1\right| \lesssim 10^{-27}\right)$; both of these features are crucial to understanding structure formation and origin of life; they are both simply explained, however, by inflation and quantum theory; and last but not least, (vii) The existence of the universal gravitational "force", crucial to the evolution of the universe leading to structure formation, which is dictated by string theory. ${ }^{\mathrm{t}}$

These provide only a sample of examples; some of them involve very small numbers like $\left(Q_{e^{-}} / Q_{p}+1\right) \lesssim 10^{-19}, m\left(\nu_{3}\right) / m_{\text {top }} \sim 10^{-12}, Y_{B} \sim 10^{-10}$, and $\left|\Omega\left(t_{E W}\right)-1\right| \lesssim$ $10^{-27}$. Each of these plays a crucial or an important role in processes leading to the evolution of life as we know it. Thus they are somehow "needed" by (or are at least compatible with) anthropic reasoning. At the same time, each of these has a beautiful explanation in terms of an underlying microscopic theory. ${ }^{\mathrm{u}}$ These and many such examples accumulating over 400 years suggest that anthropic considerations, though clearly relevant, may well coexist with an understanding of nature in the traditional sense, at least in most cases, rather than being a substitute for it.

Based on these features, and the fact that our current understanding (including formulation) of string/M theory is rather preliminary so as not to permit a real preference for the landscape picture, I tend to lean towards the traditional approach to understanding ${ }^{\mathrm{w}} \mathrm{x}$. I believe that much can be gained by continuing to search for

\footnotetext{
${ }^{\mathrm{t}}$ The extreme weakness of the gravitational coupling $G_{N}$ or correspondingly the large hierarchy between $M_{\text {Planck }}$ and $m_{p}$ remains, however, to be explained.

${ }^{u}$ I should, however, mention that the idea of inflation remains at present as a paradigm with strong observational support. Derivation of a suitable particle particle theory model implementing this idea from an underlying theory like string theory remains a challenge.

${ }^{v}$ Here I distinguish between understanding what seems to be clearly microscopic phenomena (like the origin of the three families and the smallness of the cosmological constant) versus macroscopic ones such as the distance between the earth and the sun.

wI should add that such a preference for predictivity and traditional understanding of microscopic phenomena can still be compatible with the multiverse picture and anthropism as long as the set of vacuum solutions of the underlying theory (be it either a dense discretum or a selective non-dense discrete set or a sum of both, see text) contain solution(s) matching our own world.

${ }^{\mathrm{x}}$ On another note, regardless of how our sub-universe was chosen (e.g. either statistically from a large ensemble of a dense discretum or a very selective discrete set), it is a remarkable fact that, so far, at least a very large number of its features, though anthropically needed, have been amenable to understanding in the traditional sense, as exemplified above. This has been possible only in terms of a few elegant principles, some weird laws (like quantum mechanics), and a few discrete choices. Even these (principles, laws and discrete choices) which may vary from one sub-universe to another, may find a rationale within an underlying theory. Since we cannot ever tell if we have reached a dead-end in finding such principles and weird laws and thereby a dead-end in the path of understanding, unless and until the set of unexplainables (including the origin of families and the
} 
an underlying predictive theory that includes quantum gravity.

It is of course possible that the underlying theory may in general yield multiple solutions rather than a much desired unique or almost unique one, for its ground state. Even so, one needs to ascertain (a) whether, in a more final picture, ${ }^{\mathrm{y}}$ these solutions constitute only a "dense discretum" [58 - i.e. an almost continuum - or a selective (non-dense) discrete set, or possibly a sum of both, and most important, (b) whether they at least contain a sub-set of solutions (however small) matching our own world, as regards its known properties. ${ }^{\mathrm{z}}$ Until this latter feature emerges, it seems to me that the underlying theory would remain questionable and the landscape or statistically-based anthropic reasoning would not be adequate anyway in accounting for our sub-universe. But if such a feature does emerge, that in itself would be a monumental achievement. Depending upon the nature of the solutions, for instance if changing just one or a few parameters of compactification would alter some or most of the observed parameters so that the latter would not match the properties of our sub-universe, then there can still be predictivity of some or most of the observable parameters in terms of only a few. Testing these predictions would test the underlying theory. This would greatly add to our understanding.

This is the point of view amounting to a wish and a goal that I have adopted in this talk as regards the origin of an effective grand unification-like symmetry in 4D from an underlying theory. Meanwhile, regardless of the origin of such an effective symmetry, as noted in Sections 5, 6, and 8, it has ample predictions that are amenable to experimental tests in the near future, should we succeed in building the necessary facilities.

\section{Acknowledgement}

I would like to thank Kaladi S. Babu, James D. Bjorken, Savas Dimopoulos, Alon Faraggi, Shamit Kachru, Andrei Linde, Michael Peskin, John Schwarz, Ashoke Sen, Stephen Shenker, Helen Quinn, Marvin Weinstein, Frank Wilczek and Edward Witten for most helpful comments and discussions. I would also like to thank the organizers of the conference, especially Swapna Mohapatra and T. Padmanabhan, for their kind hospitality. I am grateful to Sharon Jensen for her kind patience in typing this manuscript.

cosmological constant) reduces to a null set, it seems that striving towards reducing this set is a goal that is and would remain worth pursuing, even within the multiverse picture.

${ }^{\mathrm{y}}$ In the context of string/M theory, this should at least include a non-perturbative formulation of string/M-theory, including exploration of the semi-perturbative inner region of the M-theory diagram. As alluded to in footnote s, the final picture (not yet in hand) may well and most likely will involve radical departures from the current framework.

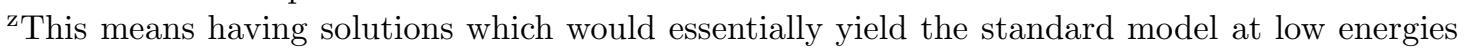
(barring entities such as neutrino masses as observed) and the desired cosmological constant. 


\section{References}

[1] S. Weinberg, Phys. Rev. Lett. 19, 1269 (1967); A. Salam, in Elementary Particle Theory Nobel Symposium, ed. by N. Svartholm (Almqvuist, Stockhold, 1968), p. 367; S. L. Glashow, Nucl. Phys. 22, 570 (1961).

[2] P. W. Higgs, Phys. Rev. Lett. 12, 132 (1964), Phys. Rev. Lett. 13, 508 (1964), Phys. Rev. 145, 1156 (1966), R. Brout and F. Englert, Phys. Rev. Lett. 13, 321 (1964), G. S. Guralnik, C. R. Hagen and T.W.G. Kibble, Phys. Rev. Lett. 13, 585 (1064).

[3] G. 't Hooft and M.J.G. Veltman, Nucl. Phys. B44, 189 (1972), Nucl. Phys. bf B50, 318 (1972).

[4] The idea of QCD evolved over a span of seven years (1965-1972). It received a decisive boost with the discovery of asymptotic freedom by D. Gross and F. Wilczek, Phys. Rev. Lett. 30, 1343 (1973); and by H. D. Politzer, Phys. Rev. Lett. 30, 1346 (1973).

[5] J. C. Pati and A. Salam, Proc. 15th High energy Conference, Batavia, reported by J. D. Bjorken, Vol. 2 p 301 (1972); Phys. Rev. 8, 1240 (1973).

[6] J.C. Pati and A. Salam, Phys. Rev. Lett. 31, 661 (1973); Phys. Rev. D10, 275 (1974).

[7] H. Georgi and S. L. Glashow, Phys. Rev. Lett. 32, 438 (1974).

[8] H. Georgi, H. Quinn and S. Weinberg, Phys. Rev. Lett. 33, 451 (1974).

[9] Y. A. Golfand and E. S. Likhtman, JETP Lett. 13, 323 (1971); J. Wess and B. Zumino, Nucl. Phys. B70, 139 (1974); D. Volkov and V. P. Akulov, JETP Lett. 16, 438 (1972).

[10] D. Friedman, S. Ferrara and P. Van Niuwenhuizen, Phys. Rev. D13, 3214 (1976); S. Deser and B. Zumino, Phys. Lett. 62B, 335 (1976); A. H. Chamseddine, R. Arnowitt and P. Nath, Phys. Rev. Lett. 49, 970 (1982).

[11] M. Green and J. H. Schwarz, Phys. Lett. 149B, 117 (1984); D. J. Gross, J. A. Harvey, E. Martinec and R. Rohm, Phys. Rev. Lett. 54, 502 (1985); P. Candelas, G. T. Horowitz, A. Strominger and E. Witten, Nucl. Phys. B258, 46 (1985).

[12] For some pioneering works on string-duality and M-theory, relevant to gauge coupling unification, see E. Witten, Nucl. Phys. B443, 85 (1995) and P. Horava and E. Witten, Nucl. Phys. B460, 506 (1996); and references therein. 
[13] A. H. Guth, Phys. Rev. D23, 347 (1981), A. D. Linde, Phys. Lett. B108, 389 (1982), A. Albrecht and P. J. Steinhardt, Phys. Rev. Lett. 48, 1220 (1982).

[14] WMAP Three Year Results: D. N. Spergel et al., astro-ph/0603449, and references therein.

[15] S. Dimopoulos, S. Raby and F. Wilczek, Phys. Rev. D24, 1681 (1981); W. Marciano and G. Senjanovic, Phys. Rev. D25, 3092 (1982) and M. Einhorn and D.R.T. Jones, Nucl. Phys. B196, 475 (1982). For work in recent years, see P. Langacker and M. Luo, Phys. Rev. D44, 817 (1991); U. Maldi, W. de Boer and H. Furtenau, Phys. Rev. Lett. B260, 131 (1991); F. Anselmo, L. Cifarelli, A. Peterman and A. Zichichi, Nuov. Cim. A104, 1817 (1991).

[16] See e.g. M. Luty, Lectures at TASI school (2002) for an overview; hep-th/0509029.

[17] J. C. Pati, Phys. Lett. B388, 532 (1996) arXiv:hep-ph/9607446.

[18] Pioneering work deriving three-family free fermionic string solutions with different effective symmetries and doublet-triplet splitting appeared in A. Faraggi, Nucl. Phys. B428, 111 (1994).

[19] H. Georgi, in Particles and Fields, Ed. by C. Carlson (AIP, NY, 1975), p.575; H. Fritzsch and P. Minkowski, Ann. Phys. 93, 193 (1975).

[20] F. Gursey, P. Ramond and R. Slansky, Phys. Lett., B60, 177 (1976); Y. Achiman and B. Stech, Phys. Lett. B77, 389 (1978); Q. Shafi, Phys. Lett. B79, 301 (1978); A. deRujula, H. Georgi and S. L. Glashow, 5th Workshop on Grand Unification, edited by K. Kang et al., World Scientific, 1984, p 88.

[21] S. M. Barr, Phys. Lett. B112, 219 (1982); J. P. Derendinger, J. E. Kim and D. V. Nanopoulos, Phys. Lett. B139, 170 (1984); I. Antoniadis, J. Ellis, J. Hagelin and D. V. Nanopoulos, Phys. Lett. B194, 231 (1987).

[22] F. Gursey, P. Ramond and P. Sikivie, Phys. Lett. B60, 177 (1976).

[23] For a recent overview emphasizing the need for supersymmetric grand unification, see S. Raby, Rept. Prog. Phys. 67, 755 (2004), hep-ph/0401155

[24] Y. Fukuda et al. (Super-Kamiokande), Phys. Rev. Lett. 81, 1562 (1998), hep-ex/9807003; K. Nishikawa (K2K) Talk at Neutrino 2002, Munich, Germany.

[25] Q. R. Ahmad et al. (SNO), Phys. Rev. Lett. 81, 011301 (2002); B. T. Cleveland et al. (Homestake), Astrophys. J. 496, 505 (1998); W. Hampel et al. (GALlEX), Phys. Lett. B447, 127, (1999); J. N. Abdurashitov et al. (SAGE) (2000), astro-ph/0204245; M. Altmann et al. (GNO), Phys. Lett. B490, 16 
(2000); S. Fukuda et al. (SuperKamiokande), Phys. Lett. B539, 179 (2002). Disappearance of $\bar{\nu}_{e}$ 's produced in earth-based reactors is established by the KamLAND data: K. Eguchi et al., hep-ex/0212021.

[26] For a historical overview of theoretical calculations of expected solar neutrino flux, see J. Bahcall, astro-ph/0209080, Int. J. Mod. Phys. A18, 3761 (2003).

[27] M. Fukugita and T. Yanagida Phys. Lett. B174, 45 (1986); V. Kuzmin, V. Rubakov and M. Shaposhnikov, Phys. Lett. BM155, 36 (1985); G. Lazarides and Q. Shafi, Phys. Lett. B258, 305 (1991).

[28] For a discussion of leptogenesis, and its success, in the model to be presented here, see J. C. Pati, Phys. Rev. D68, 072002 (2003).

[29] J. C. Pati and A. Salam, Phys. Rev. D 10, 275 (1974); R. N. Mohapatra and J. C. Pati, Phys. Rev. D 11, 566 and 2558 (1975); G. Senjanovic and R. N. Mohapatra, Phys. Rev. D 12, 1502 (1975).

[30] J. C. Pati, Lecture at ICTP Trieste School (2001) and WHEPP-7, India, hep-ph/0244240 Fujihara seminar, Tsukuba, Japan (2004), hep-ph/0407220 and talke at XI International Workshop on "Neutrino Telescopes" at Venice, Italy (February 2005), hep-ph/0507307

[31] Promising string-theory solutions yielding the G(224)-symmetry in 4D have been obtained using different approaches, by a number of authors. They include: I. Antoniadis, G. Leontaris, and J. Rizos, Phys. Lett. B245, 161 (1990); G. K. Leontaris, Phys. Lett. B 372, 212 (1996), hep-ph/9601337; A. Murayama and T. Toon, Phys. Lett. B318, 298 (1993); Z. Kakushadze, Phys. Rev. D58, 101901 (1998); G. Aldazabal, L. I. Ibanez and F. Quevedo, hep-th/9909172 C. Kokorelis, hep-th/0203187, hep-th/0209202 M. Cvetic, G. Shiu, and A. M. Uranga, Phys. Rev. Lett. 87, 201801 (2001), hep-th/0107143, and Nucl. Phys. B 615, 3 (2001), hep-th/0107166 M. Cvetic and I. Papadimitriou, hep-th/0303197. R. Blumenhagen, L. Gorlich and T. Ott, hep-th/0211059. A promising class of four dimensional three-family $\mathrm{G}(224)$-string models has recently been obtained by T. Kobayashi, S. Raby, and R. J. Zhang, hep-ph/0409098. Another class of solutions leading to the G(224)-symmetry from Type II-A orientifolds with intersecting D6-branes is obtained in M. Cvetic, T. Li and T. Liu, hep-th/0403061 more recently by C. M. Chen, T. Li and D. V. Nanopoulos, hep-th/0601064 and by B. Dutta and Y. Mimura, hep-ph/0512171. For alternative attempts based on flux compactification of Type II-B string theory leading to the G(224)symmetry, see F. Marchesano and G. Shiu, hep-th/0409132 M. Cvetic and T. Liu, hep-th/0409032 M. Cvetic, T. Li and T. Liu, hep-th/0501041.

[32] P. H. Ginsparg, Phys. Lett. B197, 139 (1987); V. S. Kaplunovsky, Nucl. Phys. B307, 145 (1988) [Erratum-ibid. B382, 436 (1992)] arXiv:hep-th/9205068. 
[33] This picture has been concretely studied by P. Rastogi (to appear).

[34] E. Witten, hep-th/9602070, Nucl. Phys. B471, 135 (1996).

[35] S. Dimopoulos and F. Wilczek, report No NSF-ITP-82-07 (1981), in The Unity of Fundamental Interactions, Proc. Erice School (1981), Plenum Press (Ed. A. Zichichi); K. S. Babu and S. M. Barr, Phys. Rev. D48, 5354 (1993).

[36] S. Weinberg, Phys. Rev. Lett. 43, 1566 (1979); E. Akhmedov, Z. Berezhiani and G. Senjanovic, Phys. Rev. D47, 3245 (1993).

[37] P. Minkowski, Phys. Lett. B67, 421 (1977); M. Gell-Mann, P. Ramond and R. Slansky, in: Supergravity, eds. F. van Nieuwenhuizen and D. Freedman (Amsterdam, North Holland, 1979) p. 315; T. Yanagida, in: Workshop on the Unified Theory and Baryon Number in the Universe, eds. O. Sawada and A. Sugamoto (KEK, Tsukuba) 95 (1979); S. L. Glashow, in Quarks and Leptons, Cargése 1979, eds. M. Levy et al. (Plenum 1980) p. 707, R. N. Mohapatra and G. Senjanovic, Phys. Rev. Lett. 44, 912 (1980).

[38] K. S. Babu, J. C. Pati and F. Wilczek, Nucl. Phys. B566, 33 (2000) hep-ph/9812538.

[39] C. H. Albright and S. M. Barr, Phys. Lett. B452, 287 (1999). The AB model has evolved through a series of papers including K. S. Babu and S.M. Barr, Phys. Lett. B381, 202 (1996), C. H. Albright and S. M. Barr, Phys. Rev. D58, 013002 (1998), C. H. Albright, K. S. Babu and S. M. Barr, Phys. Rev. Lett. 81, 1167 (1998), C. H. Albright and S. M. Barr, Phys. Rev. Lett. 85, 244 (2000).

[40] T. Blazek, M. Carena, S. Raby and C. Wagner, Phys. Rev. D56, 6919 (1997); T. Blazek, S. Raby, K. Tobe, Phys. Rev. D60, 113001 (1999), Phys. Rev. D62, 055001 (2000); R. Dermisek and S. Raby, Phys. Rev. D62, 015007 (2000).

[41] K. Dienes and J. March Russell, hep-th/9604112

[42] See e.g. K. S. Babu and R. N. Mohapatra, Phys. Rev. Lett. 70, 2845 (1993); C. S. Aulakh, B. Bajc, A. Melfo, A. Rasin and G. Senjanovic, hep-ph/0004031 M. C. Chen and K. T. Mahanthappa, Phys. Rev. D62, 113007 (2000); H. S. Goh, R. N. Mohapatra and S. P. Ng, Phys. Lett. B570, 215 (2003); B. Dutta, Y. Mimura, R.N. Mohapatra, Phys. Rev. D69, 115014 (2004); M. Bando, S. Kaneko, M. Obara and M. Tanimoto, Phys. Lett. B580, 229 (2004), hep-ph/0405071; T. Fukuyama, A. Ilakovac, T. Kikuchi and S. Meljanac, hep-ph/0411282.

[43] K. S. Babu, J. C. Pati, P. Rastogi, Phys. Rev. D71, 015005 (2005) hep-ph/0410200. 
[44] L. J. Hall, V. A. Kostelecky and S. Raby, Nucl. Phys. B267, 415 (1986); R. Barbieri, L. J. Hall and A. Strumia, Nucl. Phys. B445, 219 (1995); hep-ph/9501334 and F. Borzumati and A. Masiero, Phys. Rev. Lett. 57, 961 (1986).

[45] See e.g. U. Nierste for a recent update on the CKM matrix (hep-ph/0511125) and references therein.

[46] K. S. Babu, J. C. Pati, P. Rastogi, Phys. Lett. B621 (2005) hep-ph/0502152.

[47] P. Rastogi, Phys. Rev. D72, 075002 (2005) hep-ph/0507302.

[48] H. S. Goh, R. N. Mohapatra and Siew-Phang Ng, Phys. Rev. D68, 11508 (2003).

[49] M. L. Brooks et al. [MEGA Collaboration] Phys. Rev. Lett. 83, 1521 (1999).

[50] B. Aubert et al. [BaBar Collaboration], Phys. Rev. Lett. 95, 191801 (2005).

[51] See R. Jeannerot, S. Khalil, G. Lazarides and Q. Shafi, JHEO 010, 012 (2000); hep-ph/0002151 and references therein.

[52] N. Sakai and T. Yanagida, Nucl. Phys. B197, 533 (1982); S. Weinberg, Phys. Rev. 26, 287 (1982).

[53] K. S. Babu, J. C. Pati and F. Wilczek, Phys. Lett. B423, 337 (1998).

[54] N. Tsutsui et al., CP-PACS and JLQCD Collaboration; hep-lat/0402026.

[55] K. Turznyski, hep-ph/0110282, V2.

[56] V. Lucas and S. Raby, Phys. Rev. D55, 6986 (1997); R. Dermisek, A. Mafi and S. Raby, hep-ph/0007213, V2.

[57] SuperK Collaboration: Y. Hayato, Proc. ICHEP, Vancouver (1998); M. Earl, NNN2000 Workshop, Irvine, Calif (Feb, 2000); Y. Totsuka (private comm. May, 2001); M. Vagins, Report on SuperK Results presented at WHEPP-7 meeting, Allahabad, India (January 6, 2002).

[58] Allowing for fluxes and moduli, the multiplicity of string vacuum-solutions seems to be phenomenally large (perhaps, $\gtrsim 10^{500}$ ); see R. Bousso and J. Polchinski, JHEP 0006, 006 (2000); S. Kachru, R. Kallosh, A. D. Linde and S. P. Trivedi, Phys. Rev. D68, 046005 (2003); M. R. Douglas, JHEP 0305, 046 (2003); S. K. Ashok and M. R. Douglas, JHEP 0401, 060 (2004).

[59] L. Susskind, hep-th/0302219. For an attempt at an objective evaluation of the string-landscape picture and some critical comments, see T. Banks, M. Dine and E. Gorbatov, hep-th/0309170, JHEP 0408, 058 (2004), and T. Banks, hep-th/0412129. For a recent exposition of this picture, see J. Polchinski, hep-th/0603249 
[60] J. D. Barrow and F. J. Tipler, The Anthropic Cosmological Principle (Clarendon, Oxford, 1986). The anthropic bound on the cosmological constant was obtained by S. Weinberg, Phys. Rev. Lett. 59, 2607 (1987). For earlier work along these lines see A. D. Linde, in Three Hundred Years of Gravitation (Eds. S. Hawking and W. Israel, Cambridge University Press, 1987). For a very interesting discussion (that raises some philosophical issues) on the inflationary multiverse picture and its link with anthropism, see A. D. Linde, hep-th/0211048. For some recent expositions of related issues see e.g. M. Tegmark, A. Aguirre, M. Rees and F. Wilczek, astro-ph/0511774 and F. Wilczek, hep-ph/0512187. 